\title{
Matroids in which every pair of elements belongs to both a 4-circuit and a 4-cocircuit
}

Joel Miller

\section{VICTORIA UNIVERSITY OF WELLINGTON \\ Te Whare Wānanga o te Ūpoko o te Ika a Māui}

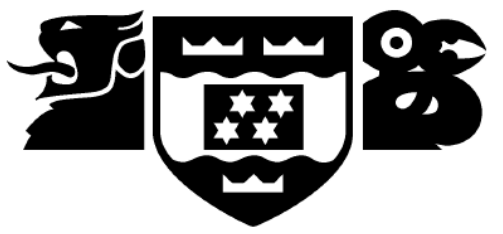

School of Mathematics, Statistics and Operations Research

Te Kura Mātai Tatauranga, Rangahau Pūnaha

A thesis

submitted to the Victoria University of Wellington in fulfilment of the requirements for the degree of

Master of Science

in Mathematics.

Victoria University of Wellington 


\begin{abstract}
In this thesis, we analyse the matroids which have the property that every pair of elements belongs to both a 4-circuit and a 4-cocircuit. In particular, we show that if a matroid with this property has at least 13 elements, then it is a spike. We also study the matroids with fewer than 13 element that have this property.
\end{abstract}




\section{Acknowledgements}

I would like to thank Geoff Whittle for his advice, supervision and encouragement, while undertaking this research and writing this thesis. 


\section{Contents}

1 Introduction 1

2 Preliminaries $\quad 3$

2.1 Circuits, cocircuits, stars and spikes . . . . . . . . . . 3

2.2 Connectivity and cones . . . . . . . . . . . . . . 6

3 The Main Theorem 13 


\section{List of Figures}

3.1 A geometric representation of $M_{1} \ldots \ldots \ldots$. . . . . . 24

3.2 A geometric representation of $M_{2} \ldots \ldots \ldots$. . . . . . . 24

3.3 A geometric representation of $M_{3} \ldots \ldots \ldots$. . . . . . . 40

3.4 A geometric representation of $M_{3}^{-8} \ldots \ldots$. . . . . . . . 40

3.5 A geometric representation of $M_{4} \ldots \ldots \ldots$. . . . . . . . . 41

3.6 A geometric representation of $M_{4}^{-4} \ldots \ldots \ldots$. . . . . . . . 41

3.7 A geometric representation of $M_{5} \ldots \ldots \ldots$. . . . . . . 51 


\section{Chapter 1}

\section{Introduction}

When writing inductive arguments about classes of 3-connected matroids, we wish to find elements to delete or contract while remaining 3-connected. When there are no such elements, the induction fails and we have to deal with the exceptional cases.

Tutte's Wheels-and-Whirls Theorem [7] shows that if we cannot find a single element to delete or contract while maintaining 3-connectivity, then the matroid is a wheel or a whirl. In other words there are only very specific exceptional cases. Seymour's Splitter Theorem [5] extends this result by showing that we can even choose the element to delete or contract such that the matroid maintains a fixed minor.

Sometimes, we wish to find a pair of elements to delete or contract while remaining 3-connected. An obvious situation that would lead to exceptional cases here, is when every pair of elements of the matroid belongs to both a 4-circuit and a 4-cocircuit.

To see this, let $M$ be any matroid containing at least 6 elements and with the property that every pair of elements belongs to both a 4-circuit and a 4cocircuit. Let $\{x, y\}$ be any pair of elements in $M$. There must be a 4 -circuit $C$ and a 4 -cocircuit $R$ both containing the pair $\{x, y\}$. This means that $M \backslash x, y$ contains the series pair $R-\{x, y\}$ and $M / x, y$ contains the parallel pair $C-\{x, y\}$. Series and parallel pairs are 2-separating and both $M \backslash x, y$ and $M / x, y$ contain at least 4 elements. Hence neither $M \backslash x, y$ nor $M / x, y$ can be 3 -connected for any pair $\{x, y\}$ in $M$. Therefore the matroids with this property are not among the matroids that remain 3-connected when we delete or contract a pair of elements. 
Jim Geelen (private communication) has stated that a much shorter proof of Rota's Conjecture for GF(4) (Geelen, Gerards and Kapoor [1]) is possible if we can easily deal with the matroids with this property. The potential to eliminate many of the exceptional cases in this and other problems motivates the main goal of this thesis. This is to determine exactly which matroids are such that every pair of elements belongs to both a 4-circuit and a 4-cocircuit.

The theorem we will prove, in the case of matroids with at least 13 elements, is as follows.

Theorem 1.1. Let $M$ be a matroid such that every pair of elements belongs to both a 4-circuit and a 4-cocircuit. If $|E(M)| \geq 13$, then $M$ is a spike.

We will also specify exactly which smaller matroids with the property are not spikes, with the exception of the 8-element, rank-4 sparse paving matroids. For the full statement of the theorem, we will need to define some of these matroids. These definitions will be given throughout Chapter 3 . Therefore we will defer the statement of the full theorem (Theorem 3.25) until the end of the Chapter 3. 


\section{Chapter 2}

\section{Preliminaries}

In this chapter we introduce some definitions, notation and basic results that will be used in this thesis. We assume that the reader is familiar with the basic concepts and terminology of matroid theory. A good introduction to matroid theory can be found in Oxley [2]. Our notation and definitions will follow [2] unless otherwise specified. In particular we will refer to some common matroids without definition. These can be found in the Appendix of $[2]$.

\subsection{Circuits, cocircuits, stars and spikes}

Unsurprisingly, circuits and cocircuits are of fundamental importance to the results in this thesis. We are particularly interested in the ways that circuits and cocircuits can interact with each other. The following well-known result is often referred to as orthogonality, and will be used freely many times throughout the thesis.

Lemma 2.1. Let $C$ be a circuit and $R$ be a cocircuit of a matroid. Then $|C \cap R| \neq 1$.

Proof. Suppose $C$ and $R$ are a circuit and a cocircuit of a matroid $M$ with $C \cap R=\{x\}$. We know that the complement of a cocircuit is a hyperplane. Hence the set $H=E(M)-R$ is a hyperplane. Clearly $x \notin H$ and $C-x \subseteq H$. But $x \in \operatorname{cl}(C-x) \subseteq \operatorname{cl}(H)=H$, a contradiction.

We will soon introduce a family of matroids called spikes. Spikes have been given several definitions in the literature. What we call a spike is 
sometimes referred to as a tipless spike. It suits us to define spikes in terms of a matroid structure which we will make use of later, stars.

Definition 2.2. Let $M$ be a matroid. For some $k \geq 3$, let the subset $A=\left\{a_{1}, b_{1}, \ldots, a_{k}, b_{k}\right\}$ of $M$ be such that $\left\{a_{i}, b_{i}, a_{j}, b_{j}\right\}$ is a circuit for all $i$ and $j$ with $1 \leq i<j \leq k$. Then the $A$ is a $k$-star of $M$, and the pairs $\left\{a_{i}, b_{i}\right\}$ are called arms of $A$. Dually, if $A$ is a $k$-star of $M^{*}$, then it is a $k$-costar of $M$.

Applying Lemma 2.1 to this definition, we get the following result. By duality we can replace cocircuit with circuit, and star with costar to get the dual result.

Lemma 2.3. If $R$ is a cocircuit containing an element $x$ in some arm of a star, then either $R$ contains both elements of that arm or $R$ contains an element from every arm of the star.

Proof. Let $A$ be a $k$-star labelled as in the definition. We may assume by relabelling that $x=a_{1}$. Suppose $R$ does not contain $b_{1}$. To avoid a single element intersection between $R$ and one of the circuits of the star, $R$ must contain an additional element from each of the 4-circuits $\left\{a_{1}, b_{1}, a_{i}, b_{i}\right\}$ for $2 \leq i \leq k$. This means that $R$ contains $a_{1}$ and one from each of $\left\{a_{i}, b_{i}\right\}$ for $2 \leq i \leq k$, an element from each arm of the star.

We now define spikes, and prove some basic facts about them.

Definition 2.4. Let $M$ be a matroid whose ground-set is both an $r$-star and an $r$-costar with the same collection of arms. Then $M$ is an $r$-spike, or spike. The arms of the star and costar are called the legs of $M$.

It is not hard to see that this definition is equivalent to Oxley's characterization of tipless spikes given in Proposition 2.1.28 of [2]. An important thing to note about $r$-spikes is that they have rank and corank equal to $r$.

Lemma 2.5. An r-spike has rank and corank $r$.

Proof. Let $M$ be an $r$-spike. Label the elements of $M$ as in Definition 2.2 relative to its underlying star. Clearly, $r\left(\left\{a_{1}, b_{1}\right\}\right) \leq 2=1+1$. Also we know that for all $i$ with $2 \leq i \leq r$ that $\left\{a_{1}, b_{1}, a_{i}, b_{i}\right\}$ is a circuit. Therefore, 
if $r\left(\left\{a_{1}, b_{1}, \ldots, a_{i-1}, b_{i-1}\right\}\right) \leq(i-1)+1=i$ then we have $b_{i} \in \operatorname{cl}\left(\left\{a_{1}, b_{1}, a_{i}\right\}\right) \subseteq$ $\operatorname{cl}\left(\left\{a_{1}, b_{1}, \ldots, a_{i-1}, b_{i-1}, a_{i}\right\}\right)$ and so

$$
\begin{aligned}
r\left(\left\{a_{1}, b_{1}, \ldots, a_{i}, b_{i}\right\}\right) & =r\left(\left\{a_{1}, b_{1}, \ldots, a_{i-1}, b_{i-1}, a_{i}\right\}\right) \\
& \leq r\left(\left\{a_{1}, b_{1}, \ldots, a_{i-1}, b_{i-1}\right\}\right)+1 \\
& \leq i+1 .
\end{aligned}
$$

Hence, inductively we know that $r\left(\left\{a_{1}, b_{1}, \ldots, a_{r-2}, b_{r-2}\right\}\right) \leq(r-2)+1=r-$ 1. But $\left\{a_{r-1}, b_{r-1}, a_{r}, b_{r}\right\}$ is a 4 -cocircuit and so $E(M)-\left\{a_{r-1}, b_{r-1}, a_{r}, b_{r}\right\}=$ $\left\{a_{1}, b_{1}, \ldots, a_{r-2}, b_{r-2}\right\}$ is a hyperplane. Therefore $r(M) \leq r$. But by duality $r^{*}(M) \leq r$. We know that $r(M)+r^{*}(M)=|E(M)|=2 r$, so we must have $r(M)=r^{*}(M)=r$.

We now show which subsets of a spike may be circuits and, by duality of the spike definition, cocircuits.

Lemma 2.6. Let $C$ be a circuit of an $r$-spike. Then $C$ is either the union of two legs, a circuit-hyperplane containing exactly one element from each leg, or a spanning circuit containing both elements from one leg and exactly one element from every other leg.

Proof. Let $M$ be an $r$-spike labelled as in Definition 2.2 relative to its underlying star and costar. Let $C$ be a nonspanning circuit of $M$. Circuits are nonempty, so we can assume by relabelling that $a_{1} \in C$.

Suppose $b_{1} \in C$. The leg $\left\{a_{1}, b_{1}\right\}$ is properly contained in many circuits, so cannot be a circuit itself. Therefore we may assume by relabelling that $a_{2} \in$ $C$. The set $\left\{a_{1}, b_{1}, a_{2}\right\}$ is still properly contained in the circuit $\left\{a_{1}, b_{1}, a_{2}, b_{2}\right\}$, so $C$ must still contain an additional element. If $b_{2} \in C$ then $C$ contains the circuit $\left\{a_{1}, b_{1}, a_{2}, b_{2}\right\}$, so must be equal to that circuit. This circuit is the union of two legs so we are done. Otherwise the dual of Lemma 2.3 tells us that $C$ must contain an element of every leg. Already we must have $|C| \geq r+1$. Hence $C$ is a spanning circuit and can contain no other elements.

Now we know that $C$ contains at most one element of every leg. But then by the dual of Lemma 2.3, $C$ must contain an element of every leg. Therefore $C$ contains exactly one element from every leg. By relabelling we can assume that $C=\left\{a_{1}, \ldots a_{r}\right\}$. Assume for contradiction that $C$ is not a hyperplane. Clearly $r(C)=|C|-1=r-1=r(M)-1$. Hence 
we can assume by relabelling that $b_{1} \in \operatorname{cl}(C)$. But then by the circuits of the form $\left\{a_{1}, b_{1}, a_{i}, b_{i}\right\}$, every $b_{i}$ is in $\operatorname{cl}(C)$. Therefore $r(C)=r(M)=r$, a contradiction.

From here it is easy to show that spikes are self-dual. To see this, we note that a matroid of fixed rank is uniquely determined by its set of nonspanning circuits [3].

Lemma 2.7. If $M$ is an $r$-spike then $M$ is self-dual, with the map $\psi$, which swaps the elements of each leg, an isomorphism between $M$ and $M^{*}$.

Proof. Let $M$ be an $r$-spike. Clearly $M^{*}$ is also an $r$-spike from the definition of a spike.

If $C$ is a 4 -circuit of $M$, then it is the union of two legs. Therefore $\psi(C)=C$. We know that $C$ is also a 4 -cocircuit of $M$, so a 4-circuit of $M^{*}$. Conversely, if $C$ is a 4-circuit of $M^{*}$, then $\psi(C)=C$ is a 4-circuit of $M$.

If $C$ is a circuit-hyperplane of $M$, then it consists of exactly one element from each leg. Therefore $\psi(C)=E(M)-C$. The complement of a circuithyperplane is a cocircuit-cohyperplane, so $E(M)-C$ is a circuit-hyperplane of $M^{*}$. Conversely, if $C$ is a circuit-hyperplane of $M^{*}$, then $\psi(C)=E(M)-C$ is a circuit-hyperplane of $M$.

We have now considered all nonspanning circuits of $M$ and $M^{*}$. Since $M$ and $\psi\left(M^{*}\right)$ have the same rank and same set of nonspanning circuits, they are equal.

\subsection{Connectivity and cones}

We now give some definitions and results relating to connectivity.

Definition 2.8. Let $M$ be a matroid and $X$ a set of elements of $M$. Let

$$
\lambda_{M}(X)=r(X)+r(E(M)-X)-r(M) .
$$

We call $\lambda_{M}$ the connectivity function of $M$. Where it is unambiguous as to which matroid we are referring, we will omit its label.

It is clear from this definition that $\lambda_{M}(E(M)-X)=\lambda_{M}(X)$. The following well-known lemma gives an alternate way of computing the connectivity function. It is a restatement of Lemma 8.1.4 from [2]. We omit the proof. 
Lemma 2.9. Let $M$ be a matroid and $X$ a set of elements of $M$. Then

$$
\lambda_{M}(X)=r(X)+r^{*}(X)-|X|
$$

Corollary 2.10. Let $M$ be a matroid and $X$ a set of elements of $M$. Then

$$
\lambda_{M}(X)=\lambda_{M^{*}}(X)
$$

We now define separations and connectedness.

Definition 2.11. Let $M$ be a matroid and $X$ a set of elements of $M$. If $\lambda_{M}(X)<k$, then the set $X$ is said to be $k$-separating. If we also have $|X|,|E(M)-X| \geq k$, then we say that the partition $(X, E(M)-X)$ is a $k$-separation. If $M$ has no $k$-separations for $k<n$, then $M$ is $n$-connected.

Some good examples which will be useful later are that parallel and series pairs are 2-separating, and triangles and triads are 3-separating. Also, we call a 4-element subset of a matroid a quad if it is both a 4-circuit and a 4-cocircuit. It is easy to see that quads are 3-separating.

Now we consider elements which appear in the closures of both sides of a separation.

Lemma 2.12. Let $(A, B)$ be a k-separation of a matroid $M$. Then

$$
r(\operatorname{cl}(A) \cap \operatorname{cl}(B))<k .
$$

Proof. By the submodularity of the rank function

$$
\begin{aligned}
r(\operatorname{cl}(A) \cap \operatorname{cl}(B)) & \leq r(\operatorname{cl}(A))+r(\operatorname{cl}(B))-r(\operatorname{cl}(A) \cup \operatorname{cl}(B)) \\
& =r(A)+r(B)-r(M) \\
& =\lambda(A) \\
& <k .
\end{aligned}
$$

In addition to connectivity, we also use the related notion of local connectivity.

Definition 2.13. Let $M$ be a matroid and $X$ and $Y$ sets of elements of $M$. Let

$$
\sqcap_{M}(X, Y)=r(X)+r(Y)-r(X \cup Y)
$$


We call $\sqcap_{M}$ the local connectivity function of $M$, and $\sqcap_{M}(X, Y)$ the local connectivity between $X$ and $Y$ in $M$. Where it is unambiguous as to which matroid we are referring, we will omit its label.

It is clear from this definition that $\sqcap(X, E(M)-X)=\lambda(X)$. The following is a basic property of $\sqcap$. It is a restatement of Lemma 8.2.3 from [2]. We omit the proof.

Lemma 2.14. Let $A$ and $B$ be sets of elements of a matroid $M$, let $A^{\prime} \subseteq A$ and let $B^{\prime} \subseteq B$. Then

$$
\sqcap\left(A^{\prime}, B^{\prime}\right) \leq \sqcap(A, B)
$$

The following lemma gives some useful inequalities on the local connectivity function. Similar results exist in the literature. We give our own proofs for these particular inequalities.

Lemma 2.15. Let $A$ and $B$ be disjoint sets of elements of a matroid $M$, let $A^{\prime}$ and $A^{\prime \prime}$ be disjoint subsets of $A$, and let $B^{\prime} \subseteq B$. Then

$$
\sqcap\left(A, B^{\prime}\right)+\sqcap\left(A^{\prime}, B\right) \leq \sqcap(A, B)+\sqcap\left(A^{\prime}, B^{\prime}\right)
$$

and

$$
\sqcap\left(A^{\prime}, B\right)+\sqcap\left(A^{\prime \prime}, B\right) \leq \sqcap(A, B)+\sqcap\left(A^{\prime}, A^{\prime \prime}\right) .
$$

Proof. By the submodularity property of the rank function, we know that

$$
\begin{aligned}
r\left(A \cup B^{\prime}\right)+r\left(A^{\prime} \cup B\right) & \geq r\left(\left(A \cup B^{\prime}\right) \cup\left(A^{\prime} \cup B\right)\right)+r\left(\left(A \cup B^{\prime}\right) \cap\left(A^{\prime} \cup B\right)\right. \\
& =r(A \cup B)+r\left(A^{\prime} \cup B^{\prime}\right) .
\end{aligned}
$$

Therefore

$$
\begin{aligned}
\sqcap\left(A, B^{\prime}\right)+\sqcap\left(A^{\prime}, B\right) & =r(A)+r\left(B^{\prime}\right)-r\left(A \cup B^{\prime}\right)+r\left(A^{\prime}\right)+r(B)-r\left(A^{\prime} \cup B\right) \\
& =r(A)+r\left(B^{\prime}\right)+r\left(A^{\prime}\right)+r(B)-\left[r\left(A \cup B^{\prime}\right)+r\left(A^{\prime} \cup B\right)\right] \\
& \leq r(A)+r\left(B^{\prime}\right)+r\left(A^{\prime}\right)+r(B)-\left[r(A \cup B)+r\left(A^{\prime} \cup B^{\prime}\right)\right] \\
& =r(A)+r(B)-r(A \cup B)+r\left(A^{\prime}\right)+r\left(B^{\prime}\right)-r\left(A^{\prime} \cup B^{\prime}\right) \\
& =\sqcap(A, B)+\sqcap\left(A^{\prime}, B^{\prime}\right),
\end{aligned}
$$

completing the proof of (2.1). Again by the submodularity property of the 
rank function, we know that

$$
\begin{aligned}
r\left(A^{\prime} \cup B\right)+r\left(A^{\prime \prime} \cup B\right)-r(B) \geq & r\left(\left(A^{\prime} \cup B\right) \cup\left(A^{\prime \prime} \cup B\right)\right) \\
& +r\left(\left(A^{\prime} \cup B\right) \cap\left(A^{\prime \prime} \cup B\right)-r(B)\right. \\
= & r\left(A^{\prime} \cup A^{\prime \prime} \cup B\right)+r(B)-r(B) \\
= & r\left(A^{\prime} \cup A^{\prime \prime} \cup B\right)+r(A)-r(A) \\
\geq & r\left(\left(A^{\prime} \cup A^{\prime \prime} \cup B\right) \cup A\right) \\
& +r\left(\left(A^{\prime} \cup A^{\prime \prime} \cup B\right) \cap A\right)-r(A) \\
= & r(A \cup B)+r\left(A^{\prime} \cup A^{\prime \prime}\right)-r(A) .
\end{aligned}
$$

Therefore

$$
\begin{aligned}
\sqcap\left(A^{\prime}, B\right)+\sqcap\left(A^{\prime \prime}, B\right) & =r\left(A^{\prime}\right)+r(B)-r\left(A^{\prime} \cup B\right)+r\left(A^{\prime \prime}\right)+r(B)-r\left(A^{\prime \prime} \cup B\right) \\
& =r\left(A^{\prime}\right)+r\left(A^{\prime \prime}\right)+r(B)-\left[r\left(A^{\prime} \cup B\right)+r\left(A^{\prime \prime} \cup B\right)-r(B)\right] \\
& \leq r\left(A^{\prime}\right)+r\left(A^{\prime \prime}\right)+r(B)-\left[r(A \cup B)+r\left(A^{\prime} \cup A^{\prime \prime}\right)-r(A)\right] \\
& =r(A)+r(B)-r(A \cup B)+r\left(A^{\prime}\right)+r\left(A^{\prime \prime}\right)-r\left(A^{\prime} \cup A^{\prime \prime}\right) \\
& =\sqcap(A, B)+\sqcap\left(A^{\prime}, A^{\prime \prime}\right) .
\end{aligned}
$$

We now describe a convenient construction which is, in some sense, the span of a point relative to a separation, or part of a separation.

Definition 2.16. Let $(A, B)$ be a partition of the ground-set of a matroid $M$. Let $a$ be some element in $A$ and let $B^{\prime} \subseteq B$ be such that $\sqcap\left(A, B^{\prime}\right) \geq 1$. Then the cone of $a$ in $(A, B)$ opposite $B^{\prime}$ is

$$
C_{(A, B)}\left(a, B^{\prime}\right)=\operatorname{cl}\left(B^{\prime} \cup a\right) \cap \operatorname{cl}(A) .
$$

If $B^{\prime}=B$, then we simply call this the cone of $a$ in $(A, B)$, or $C_{(A, B)}(a)$.

Lemma 2.17. $C_{(A, B)}\left(a, B^{\prime}\right)$ is a flat of rank at most $\sqcap\left(A, B^{\prime}\right)+1$.

Proof. $C_{(A, B)}\left(a, B^{\prime}\right)$ is an intersection of two flats so it must itself be a flat. Now suppose for contradiction that $A^{\prime}$ is an independent subset of 
$C_{(A, B)}\left(a, B^{\prime}\right)$ of size $\left|A^{\prime}\right|=\sqcap\left(A, B^{\prime}\right)+2$. So $A^{\prime} \subseteq \operatorname{cl}\left(B^{\prime} \cup a\right) \cap \operatorname{cl}(A)$, but then

$$
\begin{aligned}
\sqcap\left(A^{\prime}, B^{\prime}\right) & =r\left(A^{\prime}\right)+r\left(B^{\prime}\right)-r\left(A^{\prime} \cup B^{\prime}\right) \\
& \geq r\left(A^{\prime}\right)+r\left(B^{\prime}\right)-r\left(A^{\prime} \cup B^{\prime} \cup a\right) \\
& =r\left(A^{\prime}\right)+r\left(B^{\prime}\right)-r\left(B^{\prime} \cup a\right) \\
& \geq r\left(A^{\prime}\right)+r\left(B^{\prime}\right)-\left[r\left(B^{\prime}\right)+1\right] \\
& =\sqcap\left(A, B^{\prime}\right)+2+r\left(B^{\prime}\right)-r\left(B^{\prime}\right)-1 \\
& =\sqcap\left(A, B^{\prime}\right)+1 \\
& >\sqcap\left(A, B^{\prime}\right) \\
& \geq \sqcap\left(A^{\prime}, B^{\prime}\right),
\end{aligned}
$$

a contradiction.

Note that if $(A, B)$ is a $k$-separation then $\sqcap\left(A, B^{\prime}\right)+1 \leq \lambda(A)+1 \leq k$. So in this case, $r\left(C_{(A, B)}\left(a, B^{\prime}\right)\right) \leq k$.

The next result relates cones to 4 -circuits that go across a separation.

Lemma 2.18. Let $(A, B)$ be a k-separation of a simple matroid $M, a \in A$, $b \in B$ and $a, b \notin \operatorname{cl}(A) \cap \operatorname{cl}(B)$. Then $\left\{a, a^{\prime}, b, b^{\prime}\right\}$ is a 4-circuit if and only if

i) up to labelling, $a^{\prime} \in A-a$ and $b^{\prime} \in B-b$,

ii) $a^{\prime}, b^{\prime} \notin \operatorname{cl}(A) \cap \operatorname{cl}(B)$,

iii) $a^{\prime} \in C_{(A, B)}\left(a,\left\{b, b^{\prime}\right\}\right)$ and $b^{\prime} \in C_{(B, A)}\left(b,\left\{a, a^{\prime}\right\}\right)$, or $a^{\prime} \in C_{(A, B)}(a)$ and $b^{\prime} \in C_{(B, A)}(b)$ if $k=2$.

Also, when in fact $\left\{a, a^{\prime}, b, b^{\prime}\right\}$ is a 4-circuit, we have $C_{(A, B)}\left(a,\left\{b, b^{\prime}\right\}\right)=$ $\operatorname{cl}\left(\left\{a, a^{\prime}\right\}\right)$ and $C_{(B, A)}\left(b,\left\{a, a^{\prime}\right\}\right)=\operatorname{cl}\left(\left\{b, b^{\prime}\right\}\right)$.

Proof. Suppose $\left\{a, a^{\prime}, b, b^{\prime}\right\}$ is a 4 -circuit. If both $a^{\prime}, b^{\prime} \in A$, then since $b \in$ $\operatorname{cl}\left(\left\{a, a^{\prime}, b^{\prime}\right\}\right)$ we must have $b \in \operatorname{cl}(A)$, a contradiction. Similarly we can't have both $a^{\prime}, b^{\prime} \in B$. Thus, up to labelling, $a^{\prime} \in A-a$ and $b^{\prime} \in B-b$. Therefore (i) holds. Now suppose $a^{\prime} \in \operatorname{cl}(B)$. Then as before since $a \in \operatorname{cl}\left(\left\{a^{\prime}, b, b^{\prime}\right\}\right)$ we must have $a \in \operatorname{cl}(\operatorname{cl}(B))=\operatorname{cl}(B)$. This contradicts the initial assumptions. Hence $a^{\prime} \notin \operatorname{cl}(A) \cap \operatorname{cl}(B)$. Similarly $b^{\prime} \notin \operatorname{cl}(A) \cap \operatorname{cl}(B)$. Thus (ii) holds. Finally $a^{\prime} \in \operatorname{cl}\left(\left\{a, b, b^{\prime}\right\}\right)$, hence $a^{\prime} \in \operatorname{cl}\left(\left\{b, b^{\prime}\right\} \cup a\right) \cap \operatorname{cl}(A)=C_{(A, B)}\left(a,\left\{b, b^{\prime}\right\}\right) \subseteq$ $C_{(A, B)}(a)$. Similarly $b^{\prime} \in C_{(B, A)}\left(b,\left\{a, a^{\prime}\right\}\right) \subseteq C_{(B, A)}(b)$. So (iii) holds. 
Conversely, suppose $a^{\prime} \in A-a, b^{\prime} \in B-b, a^{\prime}, b^{\prime} \notin \operatorname{cl}(A) \cap \operatorname{cl}(B), a^{\prime} \in$ $C_{(A, B)}\left(a,\left\{b, b^{\prime}\right\}\right)$ and $b^{\prime} \in C_{(B, A)}\left(b,\left\{a, a^{\prime}\right\}\right)$. Then

$$
\begin{aligned}
\sqcap\left(A,\left\{b, b^{\prime}\right\}\right) & =r(A)+r\left(\left\{b, b^{\prime}\right\}\right)-r\left(A \cup\left\{b, b^{\prime}\right\}\right) \\
& =r(A)+2-[r(A)+1] \\
& =1 .
\end{aligned}
$$

Similarly, $\sqcap\left(\left\{a, a^{\prime}\right\}, B\right)=1$. If $k=2$ then by Lemma 2.15 , this means that

$$
\begin{aligned}
\sqcap(A, B)+\sqcap\left(\left\{a, a^{\prime}\right\},\left\{b, b^{\prime}\right\}\right) & \geq \sqcap\left(A,\left\{b, b^{\prime}\right\}\right)+\sqcap\left(\left\{a, a^{\prime}\right\}, B\right) \\
& =1+1 \\
& \geq \sqcap(A, B)+\sqcap(A, B) \\
& \geq \sqcap(A, B)+\sqcap\left(\left\{a, a^{\prime}\right\},\left\{b, b^{\prime}\right\}\right) .
\end{aligned}
$$

Therefore $\sqcap(A, B)=1=\sqcap\left(\left\{a, a^{\prime}\right\},\left\{b, b^{\prime}\right\}\right)$. Otherwise, we know that $a^{\prime} \in$ $\operatorname{cl}\left(\left\{b, b^{\prime}\right\} \cup a\right)$ and $a \notin \operatorname{cl}(B)$. In any case $r\left(\left\{a, a^{\prime}, b, b^{\prime}\right\}\right)=3$, but since $a, a^{\prime} \notin$ $\operatorname{cl}(B)$ and $b, b^{\prime} \notin \operatorname{cl}(A)$ every 3-element subset of $\left\{a, a^{\prime}, b, b^{\prime}\right\}$ is independent. Thus $\left\{a, a^{\prime}, b, b^{\prime}\right\}$ is a 4 -circuit.

Finally, suppose $\left\{a, a^{\prime}, b, b^{\prime}\right\}$ is in fact a 4-circuit. Clearly we have $a, a^{\prime} \in$ $\operatorname{cl}(A)$ and $a, a^{\prime} \in \operatorname{cl}\left(\left\{a, b, b^{\prime}\right\}\right)$. Therefore $a, a^{\prime} \in C_{(A, B)}\left(a,\left\{b, b^{\prime}\right\}\right)$. But $C_{(A, B)}\left(a,\left\{b, b^{\prime}\right\}\right)$ is a flat, so we must have $\operatorname{cl}\left(\left\{a, a^{\prime}\right\}\right) \subseteq C_{(A, B)}\left(a,\left\{b, b^{\prime}\right\}\right)$. Clearly $r\left(\operatorname{cl}\left(\left\{a, a^{\prime}\right\}\right)\right)=2$. However we must have

$$
\begin{aligned}
r\left(C_{(A, B)}\left(a,\left\{b, b^{\prime}\right\}\right)\right) & \leq \sqcap\left(A,\left\{b, b^{\prime}\right\}\right)+1 \\
& =r(A)+r\left(\left\{b, b^{\prime}\right\}\right)-r\left(A \cup\left\{b, b^{\prime}\right\}\right)+1 \\
& =r(A)+2-(r(A)+1)+1 \\
& =2 .
\end{aligned}
$$

Therefore $\operatorname{cl}\left(\left\{a, a^{\prime}\right\}\right)=C_{(A, B)}\left(a,\left\{b, b^{\prime}\right\}\right)$. By a similar argument we get that $\operatorname{cl}\left(\left\{b, b^{\prime}\right\}\right)=C_{(B, A)}\left(b,\left\{a, a^{\prime}\right\}\right)$.

The final lemma of this chapter shows that a 4-circuit that contains an element in the closure of both sides of a separation, must be contained entirely in the closure of one side of the separation.

Lemma 2.19. Let $(A, B)$ be a partition of the ground-set of a matroid $M$ 
and $g \in \operatorname{cl}(A) \cap \operatorname{cl}(B)$. Then if $C$ is a 4-circuit containing $g$, either $C \subseteq \operatorname{cl}(A)$ or $C \subseteq \operatorname{cl}(B)$.

Proof. Suppose $C$ is a 4-circuit containing $g$. By relabelling we may assume that two out of the three remaining elements of $C$ are contained in $A$, say $a_{1}$ and $a_{2}$. But then since $C$ is a circuit, the fourth element of $C$ must be contained in $\operatorname{cl}\left(\left\{g, a_{1}, a_{2}\right\}\right) \subseteq \operatorname{cl}(A)$. 


\section{Chapter 3}

\section{The Main Theorem}

In this chapter we present the main theorem of this thesis. Throughout this chapter we will refer to the property of having every pair of elements belonging to both a 4-circuit and a 4-cocircuit, as the 4-circuit-cocircuit property. Therefore, if $M$ is a matroid such that every pair of elements belongs to both a 4 -circuit and a 4 -cocircuit, we say that $M$ has the 4-circuit-cocircuit property.

The first part of the analysis is to establish that matroids with the 4circuit-cocircuit property are 3-connected. In fact there is one exception to this. This is the matroid $R_{6}$, which is isomorphic to the 2 -sum of two copies of $U_{2,4}$. Even though $R_{6}$ is not 3-connected, it is a 3-spike. This means that $R_{6}$ is not an exception to the main theorem. Also, it follows that $R_{6}$ is the only spike which is not 3-connected.

Proposition 3.1. Let $M$ be a matroid with the 4-circuit-cocircuit property. Then $M$ is either 3-connected or isomorphic to $R_{6}$.

Proof. $M$ must be connected, as every pair of elements belongs to a circuit. Also we note that $M$ cannot contain any parallel or series pairs, because such a pair cannot belong to a 4-circuit or a 4-cocircuit respectively. Hence $M$ is both simple and cosimple. Suppose that $M$ has a 2-separation $(A, B)$. We will show that in this case $M \cong R_{6}$.

Claim 3.1.1. $\operatorname{cl}(A) \cap \operatorname{cl}(B)=\emptyset$

Proof. Suppose there is an element $g$ of $M$ in $\operatorname{cl}(A) \cap \operatorname{cl}(B)$. In this case $g$ is the only element in $\operatorname{cl}(A) \cap \operatorname{cl}(B)$, as by Lemma $2.12 r(\operatorname{cl}(A) \cap \operatorname{cl}(B))$ has rank at most 1 and $M$ has no parallel pairs. Also, $g$ is clearly a member of 
both $C_{(A, B)}\left(a_{1}\right)$ and $C_{(B, A)}\left(b_{1}\right)$. Let $a_{1} \in A-g$ and $b_{1} \in B-g$ and let $C$ be a 4-circuit containing both $a_{1}$ and $b_{1}$. Then by Lemma $2.18, C=\left\{a_{1}, a_{1}^{\prime}, b_{1}, b_{1}^{\prime}\right\}$ where $a_{1}^{\prime} \in A-a_{1}, b_{1}^{\prime} \in B-b_{1}, a_{1}^{\prime} \neq g, b_{1}^{\prime} \neq g, a_{1}^{\prime} \in C_{(A, B)}\left(a_{1}\right), b_{1}^{\prime} \in C_{(B, A)}\left(b_{1}\right)$. Now let $G$ be a 4 -cocircuit containing both $a_{1}$ and $b_{1}$. Then $E(M)-G$ is a hyperplane. There are two cases, either $g \in E(M)-G$ or $g \in G$.

Suppose $g \in E(M)-G$. Then all other elements in one of $C_{(A, B)}\left(a_{1}\right)$ or $C_{(B, A)}\left(b_{1}\right)$ are in $G$ or else either $a_{1}$ or $b_{1}$, respectively, is in the hyperplane $E(M)-G$. We know $|G|=4$, so we must have $G=\left\{a_{1}, a_{1}^{\prime}, b_{1}, b_{1}^{\prime}\right\}=C$ and thus $C_{(A, B)}\left(a_{1}\right)=\left\{a_{1}, a_{1}^{\prime}, g\right\}$ and $C_{(B, A)}\left(b_{1}\right)=\left\{b_{1}, b_{1}^{\prime}, g\right\}$. Clearly $a_{1}, a_{1}^{\prime} \notin$ $\operatorname{cl}\left(E(M)-\left\{a_{1}, a_{1}^{\prime}, b_{1}, b_{1}^{\prime}\right\}\right)$ and therefore $a_{1}, a_{1}^{\prime} \notin \operatorname{cl}\left(A \cup g-\left\{a_{1}, a_{1}^{\prime}\right\}\right)$. So $r\left(A \cup g-\left\{a_{1}, a_{1}^{\prime}\right\}\right)<r(A \cup g)$. We know that

$$
\begin{aligned}
& r\left(A \cup g-\left\{a_{1}, a_{1}^{\prime}\right\}\right)+r(B \cup g) \\
& \quad \geq r\left(\left(A \cup g-\left\{a_{1}, a_{1}^{\prime}\right\}\right) \cap(B \cup g)\right)+r\left(\left(A \cup g-\left\{a_{1}, a_{1}^{\prime}\right\}\right) \cup(B \cup g)\right) .
\end{aligned}
$$

Hence

$$
\begin{aligned}
r\left(E(M)-\left\{a_{1}, a_{1}^{\prime}\right\}\right)= & r\left(\left(A \cup g-\left\{a_{1}, a_{1}^{\prime}\right\}\right) \cup(B \cup g)\right) \\
\leq & r\left(A \cup g-\left\{a_{1}, a_{1}^{\prime}\right\}\right)+r(B \cup g) \\
& -r\left(\left(A \cup g-\left\{a_{1}, a_{1}^{\prime}\right\}\right) \cap(B \cup g)\right) \\
= & r\left(A \cup g-\left\{a_{1}, a_{1}^{\prime}\right\}\right)+r(B)-r(\{g\}) \\
= & r\left(A \cup g-\left\{a_{1}, a_{1}^{\prime}\right\}\right)+r(B)-1 \\
< & r(A \cup g)+r(B)-1 \\
= & r(A)+r(B)-1 \\
= & r(M)
\end{aligned}
$$

and so $\left\{a_{1}, a_{1}^{\prime}\right\}$ is a series pair, a contradiction.

Now suppose $g \in G$. Let $D$ be a 4 -circuit containing both $a_{1}$ and $g$. By Lemma 2.19, $D \subseteq \operatorname{cl}(A)$. So $D$ contains an element $a_{2} \in A-C_{(A, B)}\left(a_{1}\right)$. By finding a 4-circuit containing $a_{2}$ and $b_{1}$, there is an element $a_{2}^{\prime} \in C_{(A, B)}\left(a_{2}\right)-$ $\left\{a_{2}, g\right\}$. Similarly we have $b_{2} \in B-C_{(B, A)}\left(b_{1}\right)$ and $b_{2}^{\prime} \in C_{(B, A)}\left(b_{2}\right)-\left\{b_{2}, g\right\}$. At least one of each of $\left\{a_{2}, a_{2}^{\prime}\right\}$ and $\left\{b_{2}, b_{2}^{\prime}\right\}$ is in $G$ or else one of these sets is contained entirely in $E(M)-G$, which would mean that $g \in \operatorname{cl}(E(M)-G)$. This means that there are at least 5 elements in $G$, a contradiction. 
Therefore $\operatorname{cl}(A) \cap \operatorname{cl}(B)=\emptyset$. By duality, $\operatorname{cl}^{*}(A) \cap \mathrm{cl}^{*}(B)=\emptyset$.

Claim 3.1.2. Each cone of $(A, B)$ contains at least two elements.

Proof. Suppose $a \in A$ and $b \in B$ and let $C$ be a 4-circuit containg both $a$ and $b$. By Lemma 2.18, this circuit contains additional elements $a^{\prime} \in C_{(A, B)}(a)$ and $b^{\prime} \in C_{(B, A)}(b)$.

Let $G$ be a 4 -cocircuit containing elements $a_{1} \in A$ and $b_{1} \in B$. We know from the dual of Lemma 2.18 that $G$ must also contain elements $a_{1}^{\prime} \in A$ and $b_{1}^{\prime} \in B$. Let $H=E(M)-\left\{a_{1}, a_{1}^{\prime}, b_{1}, b_{1}^{\prime}\right\}$. Then $H$ is the complement of a cocircuit, so is a hyperplane.

Claim 3.1.3. $H$ contains at most one element from each cone.

Proof. Suppose $H$ contains two elements from a cone. Then $H$ contains an entire cone as cones in a 2-separation have rank at most 2. We may assume by relabelling that $H$ contains $C_{(A, B)}\left(a_{2}\right)$ for some $a_{2} \in A$. Then for any other element $c \notin C_{(A, B)}\left(a_{2}\right)$ in the hyperplane, the entire cone of $c$ must be in the hyperplane. To see this let $a_{2}^{\prime}$ be an additional element of $C_{(A, B)}\left(a_{2}\right)$. If $c \in B$ and we let $c^{\prime} \in C_{(B, A)}(c)-c$, then by Lemma $2.18\left\{a_{2}, a_{2}^{\prime}, c, c^{\prime}\right\}$ is a 4-circuit, and thus $c^{\prime} \in \operatorname{cl}\left(\left\{a_{2}, a_{2}^{\prime}, c\right\}\right) \subseteq \operatorname{cl}(H)$. On the other hand, suppose $c \in A$ and let $c^{\prime} \in C_{(A, B)}(c)-c$. We know that $c \notin \operatorname{cl}\left(\left\{a_{2}, a_{2}^{\prime}\right\}\right)$ so $r\left(\left\{a_{2}, a_{2}^{\prime}, c, c^{\prime}\right\}\right) \geq 3$ and hence $\sqcap\left(\left\{a_{2}, a_{2}^{\prime}\right\},\left\{c, c^{\prime}\right\}\right) \leq 1$. Also $\sqcap(A, B)=\lambda(A)=1$ and $\sqcap\left(\left\{a_{2}, a_{2}^{\prime}\right\}, B\right)=\sqcap\left(\left\{c, c^{\prime}\right\}, B\right)=1$. By Lemma 2.15 , this means that

$$
\begin{aligned}
\sqcap(A, B)+\sqcap\left(\left\{a_{2}, a_{2}^{\prime}\right\},\left\{c, c^{\prime}\right\}\right) & \geq \sqcap\left(\left\{a_{2}, a_{2}^{\prime}\right\}, B\right)+\sqcap\left(\left\{c, c^{\prime}\right\}, B\right) \\
& =1+1 \\
& =\sqcap(A, B)+1 \\
& \geq \sqcap(A, B)+\sqcap\left(\left\{a_{2}, a_{2}^{\prime}\right\},\left\{c, c^{\prime}\right\}\right) .
\end{aligned}
$$

Therefore $\Pi\left(\left\{a_{2}, a_{2}^{\prime}\right\},\left\{c, c^{\prime}\right\}\right)=1$ which means that $\left\{a_{2}, a_{2}^{\prime}, c, c^{\prime}\right\}$ is a circuit and thus $c^{\prime} \in \operatorname{cl}\left(\left\{a_{2}, a_{2}^{\prime}, c\right\}\right) \subseteq \operatorname{cl}(H)$. We can see that in either case the entire cone of $c$ is in the hyperplane. This means that we must have the whole of the cones of $a_{1}, a_{1}^{\prime}, b_{1}$ and $b_{1}^{\prime}$ contained in $\left\{a_{1}, a_{1}^{\prime}, b_{1}, b_{1}\right\}$. Thus $C_{(A, B)}\left(a_{1}\right)=$ $\left\{a_{1}, a_{1}^{\prime}\right\}$ and $C_{(B, A)}\left(b_{1}\right)=\left\{b_{1}, b_{1}^{\prime}\right\}$. Clearly $a_{1}, a_{1}^{\prime} \notin \operatorname{cl}\left(E(M)-\left\{a_{1}, a_{1}^{\prime}, b_{1}, b_{1}^{\prime}\right\}\right)$ 
and therefore $a_{1}, a_{1}^{\prime} \notin \operatorname{cl}\left(A-\left\{a_{1}, a_{1}^{\prime}\right\}\right)$. So $r\left(A-\left\{a_{1}, a_{1}^{\prime}\right\}\right)<r(A)$. We know that

$$
1 \geq \sqcap\left(A-\left\{a_{1}, a_{1}^{\prime}\right\}, B\right) \geq \sqcap\left(\left\{a_{2}, a_{2}^{\prime}\right\},\left\{b_{1}, b_{1}^{\prime}\right\}\right)=1 .
$$

Hence

$$
\begin{aligned}
r\left(E(M)-\left\{a_{1}, a_{1}^{\prime}\right\}\right) & =r\left(\left(A-\left\{a_{1}, a_{1}^{\prime}\right\}\right) \cup B\right) \\
& =r\left(A-\left\{a_{1}, a_{1}^{\prime}\right\}\right)+r(B)-1 \\
& <r(A)+r(B)-1 \\
& =r(M)
\end{aligned}
$$

and so $\left\{a_{1}, a_{1}^{\prime}\right\}$ is a series pair, a contradiction.

So $H$ doesn't contain more than one element from each cone. Equivalently, the 4-cocircuit $\left\{a_{1}, a_{1}^{\prime}, b_{1}, b_{1}^{\prime}\right\}$ misses at most one element from each cone. So $A$ contains either one or two cones. If $A$ contains two cones then $a_{1}$ must be on one and $a_{1}^{\prime}$ must be on the other. Each of these cones must contain exactly one other element. But then these cones are series pairs, a contradiction. Hence $A$ has only one cone. Similarly $B$ has only one cone. Each of these cones must contain at least three elements or else it would be a series pair. But neither can contain more than three elements or else $\left\{a_{1}, a_{1}^{\prime}, b_{1}, b_{1}^{\prime}\right\}$ would miss more than one element of a cone. So $M \cong U_{2,4} \oplus_{2} U_{2,4} \cong R_{6}$.

We now begin to restrict the maximum size that a star of a matroid $M$ with the 4-circuit-cocircuit property can be, with $M$ not being a spike.

Lemma 3.2. Let $M$ be a matroid with the 4-circuit-cocircuit property. If $M$ contains a 5-star $A$, then $M$ is a spike.

Proof. Let $\left\{a_{1}, b_{1}\right\}$ and $\left\{a_{2}, b_{2}\right\}$ be arms of $A$. Let $G$ be a 4 -cocircuit containing $a_{1}$ and $a_{2}$. Then $G$ cannot contain an element from the other arms of the star $A$. Hence every pair of arms of $A$ is a 4-cocircuit as well as being a 4-circuit, so $A$ is also a 5 -costar with the same arms.

Now suppose that $M$ contains a $k$-star $B$ for some $k \geq 5$ which is also a $k$-costar with the same arms, labelled as in Definition 2.2. If $B$ is the whole ground set of $M$, then by our definition of spikes, $M$ is a $k$-spike and we are done. Otherwise we have an additional element $x \notin B$. For each arm $\left\{a_{i}, b_{i}\right\}$ of $B$, there is a 4 -cocircuit containing $x$ and $a_{i}$, which must contain 
$b_{i}$ or else by Lemma 2.3 it would have to contain an element from each of the at least four other arms of $B$, a contradiction. The fourth element cannot belong to $B$ or else the 4 -cocircuit will intersect a circuit at a single element. Therefore the fourth element is an additional element $x_{i} \notin B \cup x$ and so for all $i$ with $1 \leq i \leq k,\left\{x, x_{i}, a_{i}, b_{i}\right\}$ is a 4 -cocircuit with the $x_{i}$ possibly not pairwise distinct. Dually, a 4 -circuit containing $x$ and $a_{1}$ must contain $b_{1}$ and no other elements of $B$, but it must also contain all of $x_{2}, x_{3}, \ldots, x_{k}$ in order not to intersect one of the above 4-cocircuits at a single element. Therefore $x_{2}=x_{3}=\cdots=x_{k}=y$ and $\left\{x, y, a_{1}, b_{1}\right\}$ is a 4 -circuit. Similarly a 4-circuit containing $x$ and $a_{2}$ must contain $b_{2}$ and must also contain $y$ and $x_{1}$. Therefore $x_{1}=y$ and $\left\{x, y, a_{2}, b_{2}\right\}$ is a 4-circuit. Finally a 4-circuit containing $x$ and $a_{i}$ for any $i$ with $1 \leq i \leq k$ must contain $b_{i}$ and $y$. Hence $B \cup\{x, y\}$ is both a $(k+1)$-star and a $(k+1)$-costar with the arms of $B$ and $\{x, y\}$ being its arms.

By induction on $k, M$ must be a spike.

In order to start putting bounds on the size of a matroid with the 4circuit-cocircuit property, we need to restrict the number of points that can occur in a rank-2 flat. The following small lemma restricts the number of points in a rank-2 flat to three. We call a rank-2 4-element set that does not contain any loops or parallel pairs a $U_{2,4}$-restriction. Note that any 3-element subset of a $U_{2,4}$-restriction is a triangle, and conversely, the union of any two triangles sharing exactly two elements is a $U_{2,4}$-restriction, provided it does not contain a parallel pair.

Lemma 3.3. Let $M$ be a matroid with the 4-circuit-cocircuit property. Then $M$ does not contain a $U_{2,4}$-restriction.

Proof. Suppose $M$ has a $U_{2,4}$-restriction $\{a, b, c, d\}$. Clearly $M \nsubseteq R_{6}$ so by Proposition 3.1, $M$ is 3-connected. A 4-circuit containing $a$ and $b$ must contain two elements not in $\operatorname{cl}(\{a, b, c, d\})$. Thus, there are at least two elements $e, f \notin \operatorname{cl}(\{a, b, c, d\})$. The 4-cocircuit containing $a$ and $e$ must contain two other elements of $\{a, b, c, d\}$ so by relabelling we can assume that $\{a, b, c, e\}$ is a 4-cocircuit. The 4-cocircuit containing $d$ and $e$ must also contain two other elements of $\{a, b, c, d\}$ so by relabelling we can assume that $\{b, c, d, e\}$ 
is a 4 -cocircuit. Therefore $a, d \in \operatorname{cl}^{*}(\{b, c, e\})$ so $r^{*}(\{a, b, c, d\}) \leq 3$. Hence

$$
\begin{aligned}
\lambda(\{a, b, c, d\}) & =r(\{a, b, c, d\})+r^{*}(\{a, b, c, d\})-|\{a, b, c, d\}| \\
& \leq 2+3-4 \\
& =1
\end{aligned}
$$

Since $M$ contains at least two elements not in $\{a, b, c, d\}$, the partition $(\{a, b, c, d\}, E(M)-\{a, b, c, d\})$ is a 2-separation. This contradicts the 3connectedness of $M$.

It is now possible to put an upper bound on the size of a non-spike matroid with the 4-circuit-cocircuit property.

Proposition 3.4. Let $M$ be matroid with the 4-circuit-cocircuit property. If $E(M) \geq 38$ then $M$ is a spike.

Proof. Let $G=\{a, b, c, d\}$ be a 4-cocircuit of $M$. If $x \notin G$ is another element of $M$, then there must be a 4 -circuit containing $x$ and either two or three elements of $G$.

Consider the collection of 4 -circuits containing $\{a, b, c\}$ and not $d$. Let $A_{1}$ be the set of elements not in $G$ belonging to one of these 4-circuits. Then $A_{1} \subseteq \operatorname{cl}(\{a, b, c\})$ so $r\left(\{a, b, c\} \cup A_{1}\right)=3$. Evidently, $a, b, c \notin \operatorname{cl}\left(A_{1}\right)$ as those elements belong to the cocircuit $G$ which is disjoint from $A_{1}$. Therefore $r\left(A_{1}\right) \leq 2$. On the other hand, Lemma 3.3 means that $M$ does not contain a $U_{2,4}$-restriction. So $\left|A_{1}\right| \leq 3$. The same applies to the sets of elements not in $G$ that we get from the other three 3 -element subsets of $G\left(A_{2}, A_{3}\right.$ and $\left.A_{4}\right)$.

Now consider the collection of 4-circuits containing $\{a, b\}$ and not $c$ and $d$. Let $B_{1}$ be the set of elements not in $G$ belonging to one of these 4circuits. Suppose we have elements $x_{1}, y_{1}, x_{2}, y_{2} \in B_{1}$ such that $\left\{a, b, x_{1}, y_{1}\right\}$ and $\left\{a, b, x_{2}, y_{2}\right\}$ are 4-circuits. Clearly

$$
\sqcap\left(\left\{x_{1}, y_{1}\right\},\{a, b\}\right)=\sqcap\left(\left\{x_{2}, y_{2}\right\},\{a, b\}\right)=1 .
$$

Since $\left\{x_{1}, y_{1}, x_{2}, y_{2}\right\}$ is contained in a hyperplane not containing $a$ and $b$, $a, b \notin \operatorname{cl}\left\{\left\{x_{1}, y_{1}, x_{2}, y_{2}\right\}\right.$. Therefore

$$
r\left(\left\{x_{1}, y_{1}, x_{2}, y_{2}, a, b\right\}\right)=r\left(\left\{x_{1}, y_{1}, x_{2}, y_{2}\right\}\right)+1
$$


Hence $\sqcap\left(\left\{x_{1}, y_{1}, x_{2}, y_{2}\right\},\{a, b\}\right)=1$. Therefore by Lemma 2.15 if $\left\{x_{1}, y_{1}\right\}$ and $\left\{x_{2}, y_{2}\right\}$ are disjoint then $\sqcap\left(\left\{x_{1}, y_{1}\right\},\left\{x_{2}, y_{2}\right\}\right) \geq 1$ meaning that

$$
r\left(\left\{x_{1}, y_{1}, x_{2}, y_{2}\right\}\right) \leq 3 .
$$

We cannot have a $U_{2,4}$-restriction, so $r\left(\left\{x_{1}, y_{1}, x_{2}, y_{2}\right\}\right)=3$, which means that $r\left(\left\{x_{1}, y_{1}, x_{2}, y_{2}, a, b\right\}\right)=4$. Suppose $\left\{x_{1}, y_{1}, x_{2}, y_{2}\right\}$ contains a triangle which we can assume by relabelling is $\left\{x_{1}, y_{1}, x_{2}\right\}$. By circuit elimination there is a circuit contained in $\left\{y_{1}, x_{2}, a, b\right\}$. We know $a, b \notin \operatorname{cl}\left(\left\{y_{1}, x_{2}\right\}\right)$ because of the 4 -cocircuit and $y_{1}, x_{2} \notin \operatorname{cl}(\{a, b\})$ because that would create triangles inside 4-circuits so $\left\{y_{1}, x_{2}, a, b\right\}$ must be a 4-circuit. Recall that $r\left(\left\{x_{1}, y_{1}, x_{2}, y_{2}, a, b\right\}\right)=4$. But $x_{1} \in \operatorname{cl}\left(\left\{y_{1}, a, b\right\}\right)$ and $y_{1} \in \operatorname{cl}\left(\left\{x_{2}, a, b\right\}\right)$ so $r\left(\left\{x_{2}, y_{2}, a, b\right\}\right)=4$, a contradiction. Therefore $\left\{x_{1}, y_{1}, x_{2}, y_{2}\right\}$ does not contain a triangle and so must be a 4 -circuit. Hence $\{a, b\},\left\{x_{1}, y_{1}\right\}$ and $\left\{x_{2}, y_{2}\right\}$ are arms of a 3 -star.

If $\left\{x_{1}, y_{1}\right\}$ and $\left\{x_{2}, y_{2}\right\}$ are not disjoint, by relabelling suppose $x_{1}=x_{2}=$ $x$, then by circuit elimination there is a circuit contained in $\left\{b, x, y_{1}, y_{2}\right\}$. We cannot have $b \notin \mathrm{cl}\left(\left\{x, y_{1}, y_{2}\right\}\right)$ so $\left\{x, y_{1}, y_{2}\right\}$ must be a triangle. Also by circuit elimination there is a circuit contained in $\left\{a, b, y_{1}, y_{2}\right\}$ which cannot contain a triangle so it must be a 4 -circuit. So overall if two pairs $\left\{x_{1}, y_{1}\right\}$ and $\left\{x_{2}, y_{2}\right\}$ form 4-circuits with $\{a, b\}$ then either the two pairs form a 3 star with $\{a, b\}$ or form a triangle whose 2-element subsets all form 4-circuits with $\{a, b\}$. If two pairs already form a triangle, then a third pair cannot also form a triangle with one of the first two pairs or else we would have a $U_{2,4}$-restriction. If a pair doesn't form a triangle with two pairs which already form a 3-star with $\{a, b\}$ then it must form a 3-star with $\{a, b\}$ and each of the other pairs, so overall we get a 4-star. Similarly an extra pair not forming triangles with the other three pairs creates a 5 -star. Now suppose that $M$ is not a spike. Then $M$ cannot contain a 5 -star, and so $\left|B_{1}\right| \leq 3+3+3=9$. The same applies to the sets of elements not in $G$ that we get from the other five 2-element subsets of $G\left(B_{2}, B_{3}, B_{4}, B_{5}\right.$ and $\left.B_{6}\right)$.

Finally, we note that each element in $E(M)-G$ must be contained in at least two of the sets among the $A_{i}$ and $B_{j}$, in order to belong to a 4 -circuit containing each of the elements of $G$. This means that

$2|E(M)-G| \leq\left|A_{1}\right|+\left|A_{2}\right|+\left|A_{3}\right|+\left|A_{4}\right|+\left|B_{1}\right|+\left|B_{2}\right|+\left|B_{3}\right|+\left|B_{4}\right|+\left|B_{5}\right|+\left|B_{6}\right|$. 
So overall, if $M$ is not a spike, then

$$
\begin{aligned}
E(M) & =|G|+|E(M)-G| \\
& \leq 4+\frac{4 \cdot 3+6 \cdot 9}{2} \\
& =4+\frac{12+54}{2} \\
& =4+33 \\
& =37 .
\end{aligned}
$$

This reduces the problem to a finite case analysis. The next few propositions get the cases for matroids with fewer than 8 elements out of the way. We remind the reader to refer the Appendix of [2] for definitions of each of the named matroids which follow.

Proposition 3.5. Let $M$ be a matroid with the 4-circuit-cocircuit property. If $|E(M)| \leq 5$ then $M$ is isomorphic to one of $U_{0,0}, U_{0,1}$ or $U_{1,1}$.

Proof. By Proposition 3.1, $M$ must be 3-connected. If $M$ has fewer than 2 elements then the property is vacuously true. If $M$ has 2 or 3 elements then $M$ contains a pair of elements but no 4 -set of elements so the property is false. Finally, if $M$ has 4 or 5 elements then both $M$ and its dual must contain a 4-circuit so have rank at least 3, contradicting the fact that the rank and corank of a matroid sum to its size.

Proposition 3.6. Let $M$ be a matroid with the 4-circuit-cocircuit property. If $|E(M)|=6$ then $M$ is a rank-3 spike (which are $R_{6}, U_{3,6}, P_{6}, Q_{6}, \mathcal{W}^{3}$ and $\left.M\left(K_{4}\right)\right)$.

Proof. By Proposition 3.1, $M$ is 3-connected or isomorphic to $R_{6}$. Both $M$ and its dual must contain a 4-circuit so have rank at least 3, hence $M$ has rank exactly 3 . The 4-circuits of $M$ are spanning, so its only non-spanning circuits are its triangles. Hence $M$ can be completely described by its triangles. By Lemma 3.3, $M$ cannot have a $U_{2,4}$-restriction Therefore every triangle of $M$ is a hyperplane so its complement is a triad. Dually, the complement of every triad of $M$ is a triangle.

If $M$ contains two disjoint triangles, then these are also triads and therefore 2-separating sets so $M$ is not 3-connected and hence $M$ is isomorphic to $R_{6}$, a rank-3 spike. 
Otherwise distinct triangles of $M$ intersect at exactly one element. Also we cannot have three triangles all intersecting at one element or else the three triangles must contain 6 other distinct elements, a contradiction. Let $E(M)=\{a, b, c, d, e, f\}$. If $M$ has no triangles then $M$ is isomorphic to the unique rank-3 matroid on 6 elements with no non-spanning circuits $U_{3,6}$, a rank-3 spike.

Otherwise $M$ has a triangle which we can assume is $\{a, b, c\}$. If $M$ has no other triangles then $M$ is isomorphic to the unique rank-3 matroid on 6 elements with a single triangle as its only non-spanning circuit $P_{6}$, a rank-3 spike.

Otherwise $M$ has an additional triangle intersecting $\{a, b, c\}$ at exactly one element, which we can assume is $\{a, d, e\}$. If $M$ has no other triangles then $M$ is isomorphic to the unique rank-3 matroid on 6 elements with two triangles intersecting at one element as its non-spanning circuits $Q_{6}$, a rank-3 spike.

Otherwise $M$ has an additional triangle intersecting both $\{a, b, c\}$ and $\{a, d, e\}$ at exactly one element. This triangle cannot contain $a$ so we can assume that it is $\{b, d, f\}$. If $M$ has no other triangles then $M$ is isomorphic to $\mathcal{W}^{3}$, a rank-3 spike.

Otherwise $M$ has an additional triangle not containing $a, b$ or $d$. Therefore this triangle must be $\{c, e, f\}$, which we can see does indeed intersect each of the other triangles at exactly one element. Every element is now in two triangles so $M$ cannot have any more triangles. Now $M$ is isomorphic to $M\left(K_{4}\right)$, a rank-3 spike.

Proposition 3.7. Let $M$ be a matroid with the 4-circuit-cocircuit property. If $|E(M)|=7$ then $M$ is isomorphic to one of $F_{7}, F_{7}^{-}, P_{7}$ or their duals.

Proof. By Proposition 3.1, $M$ must be 3-connected. By duality we may assume that $r(M)=3$. The three elements which complement any 4-cocircuit have rank 2 so must be a triangle. By Lemma 3.3, $M$ does not contain a $U_{2,4}$-restriction. This means that every triangle of $M$ is a hyperplane so the four elements which complement it form a 4-cocircuit.

Claim 3.7.1. If some 4 -cocircuit of $M$ contains a triangle, then $M \cong P_{7}$.

Proof. Let $\{a, b, c, d\}$ be a 4-cocircuit containing the triangle $\{a, b, c\}$. Then $\{e, f, g\}$ must be a triangle and $\{d, e, f, g\}$ must be a 4 -cocircuit. Let $A$ be a 
4-cocircuit containing $a$ and $e$. Then $A$ must contain another element from both triangles $\{a, b, c\}$ and $\{e, f, g\}$. By relabelling we can assume that $A$ contains $b$ and $f$ so $A=\{a, b, e, f\}$ and hence $\{c, d, g\}$ is a triangle.

Let $B$ be a 4-cocircuit containing $c$ and $g$. As before, by relabelling we can assume that $B$ contains $b$ and $f$ so $B=\{b, c, f, g\}$ and hence $\{a, d, e\}$ is a triangle.

Finally let $C$ be a 4-cocircuit containing $a$ and $g$. Then $d \notin C$ as $C$ must contain another element from each of $\{a, b, c\}$ and $\{e, f, g\}$. Also $C$ must contain another element from each of $\{a, d, e\}$ and $\{c, d, g\}$ so $C$ contains $e$ and $c$ and hence $C=\{a, c, e, g\}$ and $\{b, d, f\}$ is a triangle.

Now every pair of elements belongs to one of the 4-cocircuits identified above. Any triple of elements not already identified as a triangle must contain two elements of one of the above triangles so we cannot have any other triangles without creating a $U_{2,4}$-restriction. The unique rank 3 matroid with the above triangles as its non-spanning circuits is isomorphic to $P_{7}$.

Note that the 4-cocircuits which don't contain $d$ do not contain a triangle so they must also be spanning 4-circuits. A spanning 4-circuit containing $d$ and, without loss of generality, $a$ is $\{a, b, d, g\}$ as this does not contain a triangle. Therefore $P_{7}$ is indeed a matroid in which every pair of elements belongs to both a 4-circuit and a 4-cocircuit.

Now we know that no 4-cocircuit of $M$ contains a triangle. Let $\{a, b, c, d\}$ be a 4 -cocircuit and let $\{e, f, g\}$ be its complementary triangle. Let $A$ be a 4cocircuit containing $a$ and $f$. Then $A$ must contain exactly one more element of the triangle $\{e, f, g\}$. By relabelling we can assume that $A$ contains $g$ and $b$ so that $A=\{a, b, f, g\}$ and $\{c, d, e\}$ is a triangle.

Let $B$ be a 4-cocircuit containing $a$ and $e$. Then $B$ must contain exactly one more element of each of the triangles $\{e, f, g\}$ and $\{c, d, e\}$. By relabelling we can assume that $B$ contains $f$ and $d$ so that $B=\{a, d, e, f\}$ and $\{b, c, g\}$ is a triangle. Let $C$ be a 4 -cocircuit containing $b$ and $e$. Then $C$ must contain exactly one more element of each of the triangles $\{b, c, g\},\{c, d, e\}$ and $\{e, f, g\}$. By relabelling we can assume that $C$ contains $c$ and not $g$. Therefore $C$ contains $f$ so that $C=\{b, c, e, f\}$ and $\{a, d, g\}$ is a triangle.

Let $D$ be a 4 -cocircuit containing $d$ and $g$. Then $D$ must contain exactly one more element of each of the triangles $\{b, c, g\},\{c, d, e\}$ and $\{e, f, g\}$, and not contain $a$ or else it would contain all of the triangle $\{a, d, g\}$. By 
relabelling we can assume that $D$ contains $c$ and not $e$. Therefore $D$ contains $f$ so that $D=\{c, d, f, g\}$ and $\{a, b, e\}$ is a triangle.

Let $F$ be a 4 -cocircuit containing $e$ and $g$. Then $F$ must contain exactly one more element of each of the triangles $\{b, c, g\},\{c, d, e\},\{a, b, e\}$ and $\{a, d, g\}$, and not contain $f$ or else it would contain all of the triangle $\{e, f, g\}$. By relabelling we can assume that $F$ contains $b$ and not $a$ or $c$. Therefore $F$ contains $d$ so that $F=\{b, d, e, g\}$ and $\{a, c, f\}$ is a triangle.

Now every pair of elements belongs to one of the 4-cocircuits identified above. The unique rank-3 matroid with the above triangles as its nonspanning circuits is isomorphic to $F_{7}^{-}$. The triple $\{b, d, f\}$ is the only additional triangle we can have without creating a $U_{2,4}$-restriction. In this case the unique rank-3 matroid with the above triangles as its non-spanning circuits is isomorphic to $F_{7}$. Note that in both cases no 4-cocircuit contains a triangle so they are also spanning 4-circuits of $M$ and hence $F_{7}^{-}$and $F_{7}$ are indeed matroids in which every pair of elements belongs to both a 4-circuit and a 4-cocircuit.

At this point we need to define some matroids which are not among the common named matroids found in [2]. The following two matroids $M_{1}$ and $M_{2}$ are 8-element rank-4 matroids that have the 4-circuit-cocircuit property, but are not spikes. Both $M_{1}$ and $M_{2}$ contain one triangle, one triad and are self-dual. We have verified these properties of these matroids, and determined their collection of non-spanning circuits using the sage-matroid package [4] for Sage Mathematics Software [6]. These matroids will be used in the results that follow.

Let $M_{1}$ be the matroid represented over $\mathrm{GF}(3)$ by the following matrix.

$$
\left[\begin{array}{cccccccc}
1 & 2 & 3 & 4 & 5 & 6 & 7 & 8 \\
1 & 0 & 0 & 0 & 1 & 1 & 1 & -1 \\
0 & 1 & 0 & 0 & 1 & 1 & 0 & 1 \\
0 & 0 & 1 & 0 & 1 & 0 & 1 & 1 \\
0 & 0 & 0 & 1 & 0 & 1 & 1 & 0
\end{array}\right]
$$

A geometric representation of $M_{1}$ is given in Figure 3.1. 


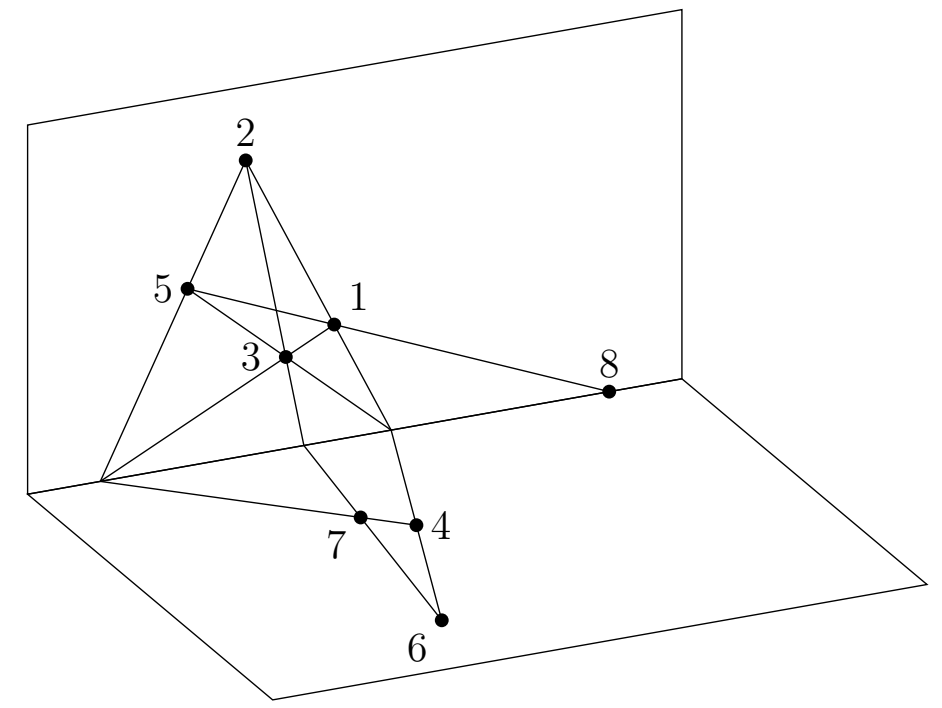

Figure 3.1: A geometric representation of $M_{1}$.

Let $M_{2}$ be the matroid represented over GF(3) by the following matrix.

$$
\left[\begin{array}{cccccccc}
1 & 2 & 3 & 4 & 5 & 6 & 7 & 8 \\
1 & 0 & 0 & 0 & 1 & 1 & 0 & 1 \\
0 & 1 & 0 & 0 & 1 & 0 & 1 & -1 \\
0 & 0 & 1 & 0 & 0 & 1 & 1 & 1 \\
0 & 0 & 0 & 1 & -1 & 1 & 1 & 1
\end{array}\right]
$$

A geometric representation of $M_{2}$ is given in Figure 3.2.

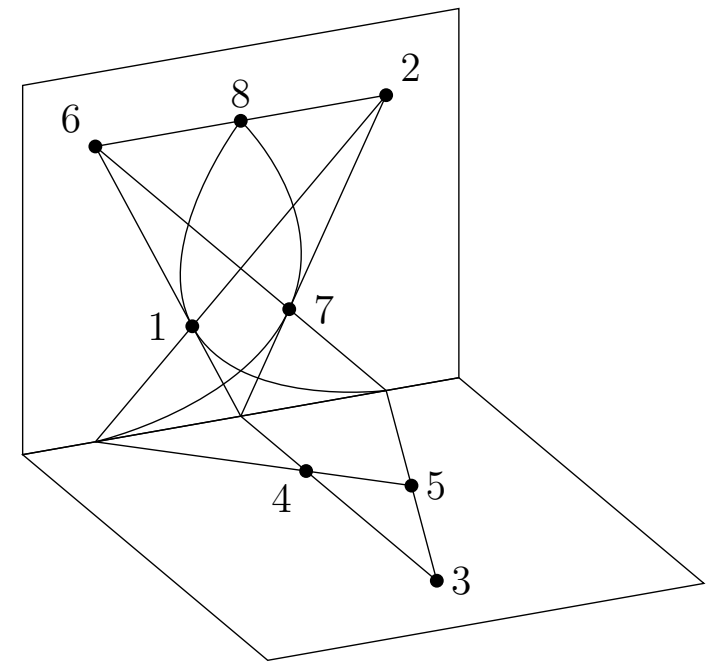

Figure 3.2: A geometric representation of $M_{2}$. 
Lemma 3.8. Let $M$ be a matroid with the 4-circuit-cocircuit property. If $|E(M)| \geq 8$ and $M$ contains a triangle or a triad then $M$ is isomorphic to one of $M\left(K_{3,3}\right), M^{*}\left(K_{3,3}\right), M_{1}$ and $M_{2}$.

Proof. By Proposition 3.1, $M$ must be 3-connected. Suppose that $M$ contains a triangle or a triad. By duality we can assume that $M$ contains a triangle $\{a, b, c\}$. There is a 4-circuit containing $a$ and $b$. A triangle cannot be contained in a 4-circuit, so there must be additional distinct elements $d$ and $e$ in this 4-circuit. We know that $c \in \operatorname{cl}(\{a, b\}) \subseteq \operatorname{cl}(\{a, b, d, e\})$ and so $r(\{a, b, c, d, e\})=3$. Note that $a, b \notin \operatorname{cl}(\{d, e\})$ but $\{c, d, e\}$ may or may not be a triangle.

Claim 3.8.1. If the set $\{a, b, c, d, e\}$ contains a 4-cocircuit, then $M \cong M_{1}$.

Proof. Suppose $\{a, b, c, d, e\}$ contains a 4 -cocircuit $R$. Then by relabelling we can assume that $a, d \in R$.

Claim 3.8.1.1. The 4-cocircuit $R$ does not contain a triangle.

Proof. Suppose $R$ contains a triangle. We may assume by relabelling that $R$ contains $\{a, b, c\}$. Therefore $R=\{a, b, c, d\}$ and $\{c, d, e\}$ may or may not be a triangle. There is a 4-cocircuit $G$ containing $c$ and $e$. In order to avoid a single element intersection between the triangle $\{a, b, c\}$ and 4 -cocircuit $G$, by relabelling we can assume that $a \in G$. If $G \subseteq\{a, b, c, d, e\}$ then $e \in \operatorname{cl}^{*}(R)$. But then

$$
\begin{aligned}
\lambda(\{a, b, c, d, e\}) & =r(\{a, b, c, d, e\})+r^{*}(\{a, b, c, d, e\})-|\{a, b, c, d, e\}| \\
& =3+3-5 \\
& =1 .
\end{aligned}
$$

Since $\{a, b, c, d, e\}$ and $E(M)-\{a, b, c, d, e\}$ both contain at least 2 elements, this contradicts the 3 -connectivity of $M$.

Therefore $G$ must contain an additional element $f$ so that $G=\{a, c, e, f\}$. This means that $f \in \mathrm{cl}^{*}(\{a, c, e\}) \subseteq \mathrm{cl}^{*}(R \cup e)$ and so $r^{*}(\{a, b, c, d, e, f\}) \leq 4$. 
Now suppose that $f \in \operatorname{cl}(\{a, b, c, d, e\})$. Then

$$
\begin{aligned}
\lambda(\{a, b, c, d, e, f\}) & =r(\{a, b, c, d, e, f\})+r^{*}(\{a, b, c, d, e, f\})-|\{a, b, c, d, e, f\}| \\
& =3+r^{*}(\{a, b, c, d, e, f\})-6 \\
& \leq 3+4-6 \\
& =1 .
\end{aligned}
$$

Since $\{a, b, c, d, e, f\}$ and $E(M)-\{a, b, c, d, e, f\}$ both contain at least 2 elements, this contradicts the 3-connectivity of $M$.

Therefore $f \notin \operatorname{cl}(\{a, b, c, d, e\})$. Now let $B$ be a 4 -circuit containing $b$ and $f$. We know that $e \in \operatorname{cl}(\{a, b, c, d\})$ and trivially $e \in \operatorname{cl}(E(M)-\{a, b, c, d\})$. So by Lemma $2.19 e \notin B$. We must have $|G \cap B| \neq 1$ and $B$ cannot contain the triangle $\{a, b, c\}$. So exactly one of $a$ and $c$ must be in $B$. We cannot have $d \in B$ or else $B$ is independent. Hence the fourth element of $B$ must be an additional element $g \in \operatorname{cl}(\{a, b, c, d, e, f\})$.

Let $H$ be a 4 -cocircuit containing $c$ and $g$. Every three element subset of $\{a, b, d, e\}$ has rank 3 so contains $c$ in its closure. This means that $H$ contains two elements of $\{a, b, d, e\}$. Therefore $g \in \mathrm{cl}^{*}(\{a, b, c, d, e\})=\mathrm{cl}^{*}(R \cup e)$. As was the case above for $f$, this means that $g \notin \operatorname{cl}(a, b, c, d, e)$. Since $\{a, b, c\}$ is a triangle, whether $a$ or $c$ is in $B,\{a, b, f, g\}$ and $\{b, c, f, g\}$ must both be 4-circuits by circuit elimination as neither set can contain a triangle, noting in particular that neither $\{a, f, g\}$ nor $\{c, f, g\}$ can be triangles in order to avoid a single-element intersection with the 4 -cocircuit $R=\{a, b, c, d\}$. Now we notice that

$$
\begin{aligned}
\lambda(\{a, b, c, d, e, f, g\})= & r(\{a, b, c, d, e, f, g\})+r^{*}(\{a, b, c, d, e, f, g\}) \\
& -|\{a, b, c, d, e, f, g\}| \\
= & 4+r^{*}(\{a, b, c, d, e, f, g\})-7 \\
\leq & 4+4-7 \\
= & 1 .
\end{aligned}
$$

So by 3-connectivity $\mid E(M)-\{a, b, c, d, e, f, g\}) \mid<2$. Therefore $|E(M)|=8$ and $M$ contains one more element $h$. Since $R=\{a, b, c, d\}$ is a 4-cocircuit, $\{e, f, g, h\}$ is a hyperplane.

By applying Lemma 2.19 to $(\{a, b, c, d\},\{e, f, g, h\})$, the 4-circuit con- 
taining $e$ and $f$ must be entirely contained within $\{e, f, g, h\}$. Therefore $h \in \operatorname{cl}(\{e, f, g\})$. Also this means that $r(M)=4$, since the hyperplane $\{e, f, g, h\}$ has rank 3 . We know $r(\{a, b, c, d, e\})=3$ so $\{f, g, h\}$ must be a triad as we cannot have coloops or series pairs. The hyperplane $\{b, d, g, h\}$ complementary to $G$ has rank 3 so $h \in \operatorname{cl}(\{b, d, g\})$.

Now consider the 4-circuit $C$ containing $a$ and $h$. We cannot have $b, c \in C$ or else either $f$ or $g$ is in $\operatorname{cl}(\{a, b, h\})$ but $g \in \operatorname{cl}(\{a, b, f\})$ so $\{d, e\}$ would be a series pair. By again applying Lemma 2.19 to $(\{a, b, c, d\},\{e, f, g, h\})$, $e \notin C$. But since $R=\{a, b, c, d\}$ and $G=\{a, c, e, f\}$ are both cocircuits $C$ must contain a second element from each. The only choices left are $d$ and $f$, so $C=\{a, d, f, h\}$. Similarly the 4-circuit containing $c$ and $h$ must be $D=\{c, d, f, h\}$. So $a, c \in \operatorname{cl}(\{d, f, h\})$. But $b, e \in \operatorname{cl}(\{a, c, d\})$ and $g \in \operatorname{cl}(\{e, f, h\})$, so $\{d, f, h\}$ spans a rank 4 matroid, a contradiction.

Now we know that $R \subseteq\{a, b, c, d, e\}$ contains $a$ and $d$ but not a triangle. Hence $R$ must contain $e$ and either $b$ or $c$. If $R$ contains $c$ then $\{c, d, e\}$ is not a triangle so we can relabel so that $R=\{a, b, d, e\}$ and $\{c, d, e\}$ may or may not be a triangle. We now know that $\{a, b, d, e\}$ is a quad, and so $(\{a, b, d, e\}, E(M)-\{a, b, d, e\})$ is an exact 3 -separation.

Let $G$ be a 4 -cocircuit containing $c$ and $d$. We cannot have $|\{a, b, c\} \cap G|=$ 1 so by relabelling we can assume that $a \in G$. If $G \subseteq\{a, b, c, d, e\}$ then $c \in \mathrm{cl}^{*}(R)$. As before, this would mean that $\lambda(\{a, b, c, d, e\})=1$, contradicting the 3 -connectivity of $M$. Therefore $G$ must contain an additional element $f$ so that $G=\{a, c, d, f\}$. This means that $f \in \mathrm{cl}^{*}(R \cup c)$ so as before if $f \in \operatorname{cl}(\{a, b, c, d, e\})$ then $\lambda(\{a, b, c, d, e, f\})=1$. This would contradict the 3 -connectivity of $M$. Therefore $f \notin \operatorname{cl}(\{a, b, c, d, e\})$.

Now let $B$ be a 4 -circuit containing $b$ and $f$. We have both $c \in \operatorname{cl}(R)$ and $c \in \operatorname{cl}(E(M)-R)$ so by Lemma $2.19 c \notin B$. We cannot have $|G \cap B|=1$ so $B$ must contain one of $a$ and $d$. Also $e \notin B$ or else $B$ is independent. Similarly we cannot have both $a, d \in B$. Hence the fourth element of $B$ must be an additional element $g \in \operatorname{cl}(\{a, b, c, d, e, f\})$.

Let $H$ be a 4-cocircuit containing $c$ and $g$. Every three element subset of $\{a, b, d, e\}$ has rank 3 so contains $c$ in its closure. This means that $H$ contains two elements of $\{a, b, d, e\}$. Therefore $g \in \mathrm{cl}^{*}(\{a, b, c, d, e\})=\mathrm{cl}^{*}(R \cup c)$. As was the case for $f$, this means that $g \notin \mathrm{cl}(\{a, b, c, d, e\})$. As before $\lambda(\{a, b, c, d, e, f, g\})=1$, so $|E(M)|=8$ and $M$ contains one more element 
$h$.

We know $R=\{a, b, d, e\}$ is a 4 -cocircuit so $\{c, f, g, h\}$ is a hyperplane. By Lemma 2.19 the 4-circuit containing $c$ and $f$ must be entirely contained within $\{c, f, g, h\}$. Therefore $\{c, f, g, h\}$ is a 4 -circuit. Also this means that $r(M)=4$, since the hyperplane $\{c, f, g, h\}$ has rank 3 . The set $\{a, b, c, d, e\}$ has rank-3. Hence it must be a hyperplane and $\{f, g, h\}$ must be a triad as we cannot have coloops or series pairs. Note that $\{f, g, h\}$ cannot also be a triangle or else it is 2-separating.

The 4-circuit containing $c$ and $d$ must be contained in $\{a, b, c, d, e\}$ by Lemma 2.19 and cannot contain both $a$ and $b$ so it must contain $e$. Therefore $\{c, d, e\}$ is not a triangle and both $\{a, c, d, e\}$ and $\{b, c, d, e\}$ must be 4-circuits. It is clear at this point that $\{a, b, c\}$ is the only triangle of $M$. The hyperplane complementary to $G$ has rank-3 and cannot contain a triangle so $\{b, e, g, h\}$ is a 4 -circuit.

Recall that the 4-circuit $B$ contains $b, f, g$ and either $a$ or $d$. If $B=$ $\{a, b, f, g\}$, then by Lemma $2.18\{c, f, g\}$ is a triangle, a contradiction. Therefore $B=\{b, d, f, g\}$. Similarly, $\{a, b, f, h\}$ and $\{a, b, g, h\}$ cannot be 4-circuits so each of $\{a, b, c, f\},\{a, b, c, g\}$ and $\{a, b, c, h\}$ is a hyperplane, so each of $\{d, e, f, g\},\{d, e, f, h\}$ and $\{d, e, g, h\}$ is a 4 -cocircuit. Also by Lemma 2.18 no other elements can be in $\operatorname{cl}(B)$ without forming a triangle. Hence $B$ is a hyperplane and $\{a, c, e, h\}$ is a 4-cocircuit. The 4-cocircuit containing $a$ and $g$ must contain at least one of $b$ and $c$, and the fourth element must also be in $\{a, b, c, d, e\}$ because of the 4-circuits $\{a, b, d, e\},\{a, c, d, e\}$ and $\{b, c, d, e\}$. But $\{c, f, g, h\}$ is a 4 -circuit so the 4 -cocircuit must also contain a second element from this. Therefore the 4-cocircuit contains $c$. The 4-cocircuit must also contain another element from each of the 4-circuits $\{b, d, f, g\}$ and $\{b, e, g, h\}$. Therefore $b$ is in the 4-cocircuit and the 4-circuit is $\{a, b, c, g\}$. This means that $\{d, e, f, h\}$ is a hyperplane not containing a triangle, so it must be a 4-circuit.

To summarise so far, $(\{a, b, d, e\},\{c, f, g, h\})$ is an exact 3-separation with $\operatorname{cl}(\{a, b, d, e\}) \cap \operatorname{cl}(\{c, f, g, h\})=\{c\},\{a, b, c\}$ is a triangle, $\{f, g, h\}$ is a triad, $\{a, b, d, e\},\{a, c, d, e\},\{b, c, d, e\},\{c, g, f, h\},\{b, d, g, f\},\{b, e, g, h\}$ and $\{d, e, f, h\}$ are 4-circuits, and $\{a, b, c, g\},\{a, b, d, e\},\{a, c, d, f\},\{a, c, e, h\}$, $\{d, e, f, g\},\{d, e, f, h\}$ and $\{d, e, g, h\}$ are 4-cocircuits. The 4-circuit containing $a$ and $g$ must contain one of $f$ and $h$, we can assume $f$ by relabelling. 
It must also contain one more from each of $\{a, b, d, e\}$ and $\{a, c, e, h\}$, so the fourth element is $e$ and the 4 -circuit is $\{a, e, f, g\}$. This must be also be a hyperplane to avoid creating additional triangles, so $\{b, c, d, h\}$ must be a 4-cocircuit. The 4-circuit containing $a$ and $h$ cannot contain $c$ by Lemma 2.19 and cannot contain $b$ or else will create a triangle. But it must contain another element of the 4-cocircuit $\{b, c, d, h\}$ so it must contain $d$. It also must contain another element of the 4-cocircuit $\{a, b, c, g\}$ so it must contain $g$. Therefore the 4-circuit is $\{a, d, g, h\}$. This must be a hyperplane or else we have another triangle, so $\{b, c, e, f\}$ is a 4 -cocircuit. At this point we can see that $M$ has the 4-circuit-cocircuit property. It is easy to check that any more 4-circuits would force another triangle. Hence we have found the complete list of non-spanning circuits so $M$ must be isomorphic to $M_{1}$ as described above and is self-dual.

We can now assume that $\{a, b, c, d, e\}$ does not contain a 4-cocircuit. Nevertheless there must be a 4 -cocircuit $R$ containing $a$ and $d$.

Claim 3.8.2. If $\{c, d, e\}$ is a triangle, then $M \cong M^{*}\left(K_{3,3}\right)$.

Proof. Suppose $\{c, d, e\}$ is a triangle. The 4-cocircuit $R$ must contain an element $x_{1}$ not in $\{a, b, c, d, e\}$ and since $|\{a, b, c\} \cap R| \neq 1$ and $|\{c, d, e\} \cap R| \neq$ $1, c \in R$. So $R=\left\{a, d, c, x_{1}\right\}$. Similarly the 4-cocircuits containing $a$ and $e, b$ and $e$, and $b$ and $d$ are $\left\{a, e, c, x_{2}\right\},\left\{b, e, c, x_{3}\right\}$ and $\left\{b, d, c, x_{4}\right\}$, where $x_{1}, x_{2}, x_{3}, x_{4} \notin\{a, b, c, d, e\}$, and the $x_{i}$ are possibly non-distinct.

Now we show that $\{a, b, c, d, e\}$ cannot contain a triad. If $\{a, b, c, d, e\}$ contains a triad containing $c$ then the triad must contain one of $a$ and $b$, and one of $d$ and $e$. But then the triad is contained in one of the 4-cocircuits listed above, a contradiction. A triad in $\{a, b, c, d\}$ must contain two elements from one of the triangles $\{a, b, c\}$ and $\{c, d, e\}$, and one from the other triangle, a contradiction. So $\{a, b, c, d, e\}$ doesn't contain a triad.

Now suppose $x_{1}=x_{2}$. Then by applying cocircuit elimination to the cocircuits $\left\{a, d, c, x_{1}\right\}$ and $\left\{a, e, c, x_{2}\right\}$ there must be cocircuit contained in $\{a, c, d, e\}$, a contradiction. So $x_{1} \neq x_{2}$. Similarly $x_{2} \neq x_{3}, x_{3} \neq x_{4}$ and $x_{4} \neq x_{1}$, though we possibly still have $x_{1}=x_{3}$ and $x_{2}=x_{4}$.

The 4-circuit $A$ containing $c$ and $a$ cannot contain $b$ but must contain a second element from both the 4-cocircuits $\left\{b, e, c, x_{3}\right\}$ and $\left\{b, d, c, x_{4}\right\}$ so $A$ must contain one of $e$ and $x_{3}$ and one of $d$ and $x_{4}$. It cannot contain both 
$d$ and $e$ or else it contains the triangle $\{c, d, e\}$. If it contains $d$ and $x_{4}$ then the rank-3 set $\left\{a, b, c, d, x_{4}\right\}$ contains the 4 -cocircuit $\left\{b, d, c, x_{4}\right\}$, so we can relabel to get into the other case. Similarly if it contains $e$ and $x_{3}$. So $A$ must contain $x_{3}$ and $x_{4}$ and hence $A=\left\{c, a, x_{3}, x_{4}\right\}$. Similarly we have 4-circuits $B=\left\{c, b, x_{1}, x_{2}\right\}, D=\left\{c, d, x_{2}, x_{4}\right\}$ and $E=\left\{c, e, x_{1}, x_{4}\right\}$.

Suppose $M$ contains an additional element $y \notin\left\{a, b, c, d, e, x_{1}, x_{2}, x_{3}, x_{4}\right\}$. Then the 4-cocircuit containing $y$ and $c$ contains one of $a$ and $b$, and one of $d$ and $e$, by relabelling suppose $a$ and $d$. But then the 4-cocircuit must also contain an additional element of $B$, a contradiction. So $E(M)=$ $\left\{a, b, c, d, e, x_{1}, x_{2}, x_{3}, x_{4}\right\}$. Therefore $8 \leq|E(M)| \leq 9$. If $|E(M)|=8$ then by relabelling we can assume that $x_{1} \neq x_{3}$ and $x_{2}=x_{4}$.

The 4-cocircuit containing $a$ and $x_{3}$ must contain either $b$ or $c$. If it contains $c$ then the fourth element must be $d$ or $e$. But then it shares exactly one element with the 4 -circuit $B=\left\{c, b, x_{1}, x_{2}\right\}$, a contradiction. So the 4cocircuit must contain $b$. The fourth element must be contained in both of the 4-circuits $B=\left\{c, b, x_{1}, x_{2}\right\}$ and $C=\left\{c, d, x_{2}, x_{3}\right\}$ but cannot be $c$, so it must be $x_{2}$ and the 4 -cocircuit must be $\left\{a, b, x_{2}, x_{3}\right\}$. But then it shares exactly one element with the 4 -circuit $E=\left\{c, e, x_{1}, x_{4}\right\}$ as $x_{4}=x_{2}$, a contradiction.

Therefore $x_{1} \neq x_{3}$ and $x_{2} \neq x_{4}$ so the $x_{i}$ are pairwise distinct, and hence $|E(M)|=9$. By applying circuit elimination to the circuits $\{a, b, c\}$ and $B=\left\{c, b, x_{1}, x_{2}\right\}$ there must be a circuit contained in $\left\{a, c, x_{1}, x_{2}\right\}$. This set shares exactly one element, $c$, with the 4-cocircuit $\left\{c, x_{3}, b, e\right\}$ so $c$ cannot be in this circuit. By 3-connectivity $\left\{a, x_{1}, x_{2}\right\}$ must be a triangle. Similarly $\left\{b, x_{3}, x_{4}\right\},\left\{d, x_{1}, x_{4}\right\}$ and $\left\{e, x_{2}, x_{3}\right\}$ are triangles. Applying circuit elimination to intersecting pairs of triangles, we must have the sets $\left\{a, e, x_{1}, x_{3}\right\}$, $\left\{a, d, x_{2}, x_{4}\right\},\left\{b, d, x_{1}, x_{3}\right\}$ and $\left\{b, e, x_{2}, x_{4}\right\}$ each containing a circuit. If we were to remove any element from any of these sets then it would share exactly one element with one of the 4-cocircuits $\left\{a, d, c, x_{1}\right\},\left\{a, e, c, x_{2}\right\},\left\{b, e, c, x_{3}\right\}$ and $\left\{b, d, c, x_{4}\right\}$, so by 3 -connectivity the four sets must all be 4-circuits.

We now have that every pair of elements of $M$ belongs to a 4 -circuit. From the 4-cocircuits we know that $E(M) \subseteq \mathrm{cl}^{*}(\{a, b, c, d, e\})$ so $r^{*}(M) \leq$ 5. Therefore $r(M) \geq 4$ and so $x_{1}, x_{2}, x_{3}, x_{4} \notin \operatorname{cl}(\{a, b, d, e\})$ otherwise the matroid would be spanned be the 4 -circuit $\{a, b, d, e\}$. So $r(M)=4$ and $\{a, b, c, d, e\}$ is a hyperplane making $\left\{x_{1}, x_{2}, x_{3}, x_{4}\right\}$ a 4 -cocircuit. Similarly any other intersection of intersecting triangles is a hyperplane and so we get 
the 4-cocircuits $\left\{x_{1}, x_{4}, a, b\right\},\left\{x_{2}, x_{3}, a, b\right\},\left\{x_{1}, x_{2}, d, e\right\}$ and $\left\{x_{3}, x_{4}, d, e\right\}$.

We now have that every pair of elements of $M$ belongs to a 4-cocircuit. Using symmetry it is now easy to show that $M$ cannot have any other nonspanning circuits than the six triangles and nine 4-circuits already identified. Hence $M$ is now uniquely determined and is isomorphic to $M^{*}\left(K_{3,3}\right)$.

Note that its dual $M\left(K_{3,3}\right)$ also has the property.

We can now assume that $\{c, d, e\}$ is not a triangle. As $\{a, b, c\}$ is a triangle, $|\{a, b, c\} \cap R| \neq 1$ so by relabelling we can assume that $b \in R$. The fourth element of $R$ cannot be contained in $\{a, b, c, d, e\}$ so there must be an additional element $f \in R$. Hence $R=\{a, b, d, f\}$ is a 4 -cocircuit. Note that $f \notin \operatorname{cl}(\{a, b, d\})$ or else $\{a, b, d, f\}$ is a 4 -circuit and $\{a, b, c, d, f\}$ contains the 4 -cocircuit $R$ so we can relabel to be in the other case. There is a 4-cocircuit $G$ containing $c$ and $e$. By relabelling we can assume that $b \in R$. The fourth element must be $g \notin\{a, b, c, d, e\}$, possibly equal to $f$, so $G=\{b, c, e, g\}$. Note that $g \notin \operatorname{cl}(\{b, c, e\})$ or else $\{b, c, e, g\}$ is a 4 -circuit and $\{a, b, c, e, g\}$ contains the 4-cocircuit $G$ so we can relabel to be in the other case.

Claim 3.8.3. If $g=f$, then $M \cong M_{2}$.

Proof. Suppose $g=f$. Then by cocircuit elimination there is a cocircuit contained in $\{a, b, c, d, e\}$. Any triad would mean that some element in only one of the two 4-cocircuits is in the coclosure of the other 4-circuit, which would mean all six elements are in the coclosure of one of the 4-cocircuits. Hence

$$
\begin{aligned}
\lambda(\{a, b, c, d, e\}) & =r(\{a, b, c, d, e\})+r^{*}(\{a, b, c, d, e\})-|\{a, b, c, d, e\}| \\
& =3+3-5 \\
& =1 .
\end{aligned}
$$

Therefore $\{a, b, c, d, e\}$ is 2-separating, a contradiction. There cannot be a 4-cocircuit contained in $\{a, b, c, d, e\}$ so it must itself be a cocircuit. There must be an element $h \notin\{a, b, c, d, e, f\}$. The 4-cocircuit containing $h$ and $b$ must contain another element from the triangle $\{a, b, c\}$, by relabelling we can assume $a$. But it must also contain another element from the 4-cocircuit $\{b, c, d, e\}$. Therefore $f, h \in \mathrm{cl}^{*}(\{a, b, c, d, e\})$ and so $r^{*}(\{a, b, c, d, e, f, h\})=$ 4. Also, a 4-circuit containing $f$ and $h$ must contain one and therefore two 
elements of the cocircuit $\{a, b, c, d, e\}$ and hence $r(\{a, b, c, d, e, f, h\})=4$. Hence

$$
\begin{aligned}
\lambda(\{a, b, c, d, e, f, h\})= & r(\{a, b, c, d, e, f, h\})+r^{*}(\{a, b, c, d, e, f, h\}) \\
& -|\{a, b, c, d, e, f, h\}| \\
= & 4+4-7 \\
= & 1 .
\end{aligned}
$$

Therefore $\{a, b, c, d, e, f, h\}$ is 2-separating so $M$ must have only one more element $i \notin\{a, b, c, d, e, f, h\}$, and $r(M)=r^{*}(M)=4$. As with $f$ and $h$, $i$ must belong to a 4-cocircuit whose other three elements are members of $\{a, b, c, d, e\}$, so $f, h, i \notin \operatorname{cl}(\{a, b, c, d, e\})$. This means that $\{a, b, c, d, e\}$ is a hyperplane and hence $\{f, h, i\}$ is a triad. Because $\{a, b, d, f\}$ and $\{b, c, e, f\}$ are both cocircuits, their complements $\{c, e, h, i\}$ and $\{a, d, h, i\}$ are hyperplanes and hence rank-3 sets. Since both $\{a, b, c, d, e\}$ and $\{f, h, i\}$ are cocircuits, the rank-3 sets cannot contain a triangle so they must be 4 -circuits. The 4-cocircuit containing $f$ and $h$ cannot contain $i$ or else it would contain the triad $\{f, h, i\}$. Both or neither of the remaining two elements may belong to the triangle $\{a, b, c\}$. It cannot be both or else we have a single element intersection with one of the 4-circuits in $\{a, b, c, d, e\}$. Therefore the 4-cocircuit contains $d$ and $e$ and is $\{d, e, f, h\}$. By the same argument the 4-cocircuit containing $f$ and $i$ is $\{d, e, f, i\}$ and the 4-cocircuit containing $h$ and $i$ is $\{d, e, h, i\}$.

The 4-circuit containing $b$ and $h$ must contain one of $f$ and $i$ but cannot contain both or else it has a single element intersection with the cocircuit $\{a, b, c, d, e\}$, so by one of the 4-cocircuits we have just listed the 4-circuit must contain $d$ or $e$. We may assume by relabelling that it contains $d$. The fourth element is either $f$ or $i$. If it is $i$ then by applying circuit elimination to this 4 -circuit and $\{a, d, h, i\}$ there must be a circuit in $\{a, b, d, h\}$. It cannot contain $h$ because of the triad $\{f, h, i\}$ but $\{a, b, d\}$ is contained in a 4 -circuit, a contradiction. So the 4-circuit is $\{b, d, h, f\}$. The complement of this rank-3 set, $\{a, c, e, i\}$, cannot contain a triad without creating a single element intersection with one of the circuits so it must be a 4-cocircuit.

The 4-circuit containing $b$ and $i$ cannot contain $h$ or else the fourth element must be contained in all of the cocircuits $\{a, b, c, d, e\},\{a, b, d, f\}$ and 
$\{b, c, e, f\}$, a contradiction. But the 4-circuit must contain an additional element from the triad $\{f, h, i\}$ so must contain $f$. The fourth element must belong to both of the 4-cocircuits $\{d, e, h, i\}$ and $\{a, c, e, i\}$ so it must be $e$ and hence $\{b, e, f, i\}$ is a 4 -circuit. The complement of this rank-3 set, $\{a, c, d, h\}$, cannot contain a triad without creating a single element intersection with one of the circuits so it must be a 4 -cocircuit.

The 4-circuit containing $a$ and $f$ must contain exactly one of $h$ or $i$ because of the triad and hyperplane $\{f, h, i\}$ so the 4-circuit must also contain either $d$ or $e$ due to the 4-cocircuit $\{d, e, h, i\}$. But it must contain another element from the 4-cocircuit $\{b, c, e, f\}$, so the 4-circuit contains $e$. Finally, the 4-cocircuit $\{a, c, d, h\}$ means that the fourth element is $h$ and so the 4circuit is $\{a, e, f, h\}$. By the same a similar argument $\{c, d, f, i\}$ is a 4 -circuit. The complements of these two rank-3 sets, $\{b, c, d, i\}$ and $\{a, b, e, h\}$, cannot contain any triads without having a single element intersection with one of the circuits so both of these sets are 4-cocircuits.

We have now identified a 4-circuit and a 4-cocircuit containing each pair of elements of $M$. It is easy to check that having any other non-spanning circuits would create a single element intersection with one of the cocircuits. Therefore the triangle $\{a, b, c\}$ and the nine 4-circuits identified above are exactly the non-spanning circuits of the rank-4 matroid $M$. Hence $M$ is uniquely determined and is isomorphic to $M_{2}$, which is self-dual.

Now we can assume that $f \neq g$. Let $x \notin\{a, b, c, d, e, f, g\}$ be an additional element of the matroid, and let $B$ be a 4 -circuit containing $b$ and $x$. Then $B$ must contain an additional element from each of the 4-cocircuits $\{a, b, d, f\}$ and $\{b, c, e, g\}$. Hence $B$ must contain one of $a, d$ and $f$, and one of $c$, $e$ and $g$. This means that $B-x \subseteq\{a, b, c, d, e, f, g\}$ and therefore $x \in$ $\operatorname{cl}(\{a, b, c, d, e, f, g\})$. Therefore $r(M) \leq 5$. The 4-cocircuit containing $x$ and $b$ must contain one of $a$ and $c$, and then another element from the 4-circuit $\{a, c, d, e\}$ so that $x$ is its only element not in $\{a, b, c, d, e\}$. This means that $x \in \mathrm{cl}^{*}(\{a, b, c, d, e\})$. Hence $r^{*}(M) \leq 5$ and $|E(M)| \leq 10$. Let $S$ be a 4-cocircuit containing $f$ and $g$. Then $S$ cannot contain an element of the triangle $\{a, b, c\}$ or else it would have to contain a second element of the triangle and still intersect one of the 4-circuits in $\{a, b, c, d, e\}$ at a single element. Hence $S$ either contains both $d$ and $e$ or neither of them, or else $S$ intersects the 4-circuit $\{a, b, d, e\}$ at a single element. 
Suppose $S$ contains neither $d$ nor $e$ so that $S=\{f, g, h, i\}$ is a 4-cocircuit for additional elements $h, i \notin\{a, b, c, d, e, f, g\}$.

Suppose $M$ contains no additional elements so that $|E(M)|=9$. Then $E(M)-S=\{a, b, c, d, e\}$ is a hyperplane. But $r(\{a, b, c, d, e\})=3$ and so $r(M)=4$ and $r^{*}(M)=5$. A 4-circuit $B$ containing $h$ and $b$ must contain one of $a, d$ and $f$, and one of $c, e$ and $g$. But it must also contain an additional element from the 4-cocircuit $S=\{f, g, h, i\}$, so one of $f$ and $g$ is in $B$. In addition, we know that there is a 4 -cocircuit containing $i, b$ and one of $\{a, c, d, e\}$, so a member of $\{a, c, d, e\}$ is in $B$. By relabelling we can assume that $f \in B$ and one of $c$ and $e$ is also in $B$. But if $c \in B$, then $B=\{b, c, f, h\}$ is a 4-circuit. Applying circuit elimination to $B$ and the triangle $\{a, b, c\},\{a, b, f, h\}$ contains a circuit. In order to avoid a single element intersection with the 4-cocircuit $G=\{b, c, e, g\}$, this circuit cannot contain $b$. Therefore $\{a, f, h\}$ is a triangle. But a 4 -cocircuit containing $i$ and $a$ must contain two elements from $\{b, c, d, e\}$. This 4-cocircuit intersects the triangle $\{a, f, g\}$ at a single element, a contradiction. Therefore $e \in B$, so that $B=\{b, e, f, h\}$ is a 4 -circuit. By symmetry, a 4-circuit containing $i$ and $b$ is either $\{b, e, f, i\}$, or $\{b, d, g, i\}$. If $\{b, e, f, i\}$ is a circuit, then by circuit elimination with $B=\{b, e, f, h\}$, there is a circuit contained in $\{e, f, h, i\}$. However this set intersects both $R=\{a, b, d, f\}$ and $G=\{b, c, e, g\}$ at a single element, so the circuit must be contained in $\{h, i\}$. This contradicts the 3-connectivity of $M$. Therefore $\{b, d, g, i\}$ is a 4 -circuit. A 4-cocircuit containing $c$ and $f$ must contain two elements from $\{a, b, d, e\}$. Suppose this 4-cocircuit contains $b$. Then it must contain an additional element of the 4-circuit $\{b, d, g, i\}$, which must be $d$. Hence $\{b, c, d, f\}$ is a 4-cocircuit. But then the set $\{e, g, h, i\}$ is disjoint from both of the 4-cocircuits $R=\{a, b, d, f\}$ and $\{b, c, d, f\}$, and so is contained in two different hyperplanes. Therefore $r(\{e, g, h, i\}) \leq 2$, so $\{e, g, h, i\}$ is a $U_{2,4}$-restriction, contradicting Lemma 3.3. Hence a 4-cocircuit containing $c$ and $f$ cannot contain $b$ so must contain $a$. The fourth element of this 4-cocircuit is either $d$ or $e$. But it must contain an additional element from the 4 -circuit $B=\{b, e, f, h\}$. Therefore $\{a, c, e, f\}$ is a 4 -cocircuit. But a 4-cocircuit containing $c$ and $e$ cannot contain $f$, or else we can relabel into the case where $g=f$.

Now suppose $M$ contains an additional element $j$ so that $|E(M)|=10$. Therefore $r(M)=r^{*}(M)=5$. Let $C$ be a 4-circuit containing $h$ and $j$. 
Suppose $i \in C$. Then exactly one of element of $C$ is in $\{a, b, c, d, e, f, g\}$. But that means that $C$ intersects one of the 4-cocircuits $R=\{a, b, d, f\}$ or $G=\{b, c, e, g\}$ at a single element, a contradiction. So $i \notin C$. But $C$ must contain an additional element of the 4 -cocircuit $S=\{f, g, h, i\}$. By relabelling we can assume that $f \in C$. The fourth element of $C$ must be an additional element of $R=\{a, b, d, f\}$, but cannot belong to $G=\{b, c, e, g\}$ or the triangle $\{a, b, c\}$ without creating a single element intersection. Hence $d \in C$, and so $C=\{d, f, h, j\}$ is a 4-circuit. A 4-cocircuit containing $h$ and $a$ must contain two elements from $\{b, c, d, e\}$. This 4-cocircuit must contain an additional element of $C=\{d, f, h, j\}$, hence the 4-cocircuit contains $d$. The fourth element must be an additional element of the triangle $\{a, b, c\}$. So the 4-cocircuit containing $h$ and $a$ must contain $d$ and either $b$ or $c$. Similarly for the 4-cocircuits containing $h$ and $b$, and $h$ and $c$. Therefore $\{a, b, c, d, h\}$ contains two different 4-cocircuits so we must have $r^{*}(\{a, b, c, d, h\})=3$. By the same process of considering 4-cocircuits containing $j$ and an element of the triangle $\{a, b, c\},\{a, b, c, d, j\}$ contains two different 4-cocircuits so we must have $r^{*}(\{a, b, c, d, j\})=3$. Therefore $j \in \mathrm{cl}^{*}(\{a, b, c, d\})$, making $r^{*}(\{a, b, c, d, h, j\})=3$. The 4-cocircuit $R=\{a, b, d, f\}$ means that $f \in \mathrm{cl}^{*}(\{a, b, d\})$. Therefore $r^{*}(\{a, b, c, d, f, h, j\})=3$. There must be a cohyperplane properly containing $\{a, b, c, d, f, h, j\}$. But then $\{e, g, i\}$ contains a circuit with two or fewer elements, contradicting the 3-connectivity of $M$.

Therefore the 4-cocircuit $S$ must contain both $d$ and $e$, so that $S=$ $\{d, e, f, g\}$. Furthermore there can be no other 4-cocircuits containing $f$ and $g$.

Suppose $|E(M)|=8$. Then there is exactly one additional element $h \notin$ $\{a, b, c, d, e, f, g\}$. We can't have $h \in \operatorname{cl}(\{a, b, c, d, e\})$ or else $\{f, g\}$ is a cocircuit, contradicting the 3-connectivity of $M$. Therefore $h \notin \operatorname{cl}(\{a, b, c, d, e\})$. This makes $\{a, b, c, d, e\}$ a hyperplane and $\{f, g, h\}$ a triad. We now know $r(M)=r^{*}(M)=4$. Let $A$ be a 4-circuit containing $h$ and $a$. Suppose $b \in A$. Then $A$ must contain an additional element of the 4-cocircuit $G=\{b, c, e, g\}$. We can't have $c \in A$ or else $A$ contains the triangle $\{a, b, c\}$. We also cannot have $e$ or $g$ in $A$ or else $A$ contains a single element of the 4-cocircuit $S=\{d, e, f, g\}$. Therefore $b \notin A$. But $A$ must still contain an additional element of the 4-cocircuit $R=\{a, b, d, f\}$. Hence one of $f$ and $d$ is in $A$. The fourth element of $A$ must be an additional element of the 
4-cocircuit $S=\{d, e, f, g\}$, but $A$ cannot contain a single element of the 4-cocircuit $G=\{b, c, e, g\}$. Hence both $d$ and $f$ are members of $A$, so that $A=\{a, d, f, h\}$ is a 4 -circuit. Similarly a 4-circuit containing $h$ and $c$ must be $\{c, e, g, h\}$. A 4-cocircuit containing $f$ and $c$ must contain two elements from $\{a, b, d, e\}$. But the 4-cocircuit must contain an additional element of the 4-circuit $\{c, e, g, h\}$. Hence $e$ is a member of the 4-cocircuit. The fourth element of the 4-cocircuit must be an additional element of the triangle $\{a, b, c\}$. But the 4-cocircuit must contain an additional element of the 4-circuit $A=\{a, d, f, h\}$ Therefore $a$ is in the 4-cocircuit, so the 4-cocircuit is $\{a, c, e, f\}$. But a 4-cocircuit containing $c$ and $e$ cannot contain $f$, or else we can relabel into the case where $g=f$.

Suppose $|E(M)|=9$. Then there are two additional element $h, i \notin$ $\{a, b, c, d, e, f, g\}$. A 4-cocircuit containing $h$ and $i$ cannot contain any element of the triangle $\{a, b, c\}$, or else it would have to contain two other members of $\{a, b, c, d, e\}$. The 4-cocircuit must contain either both of $d$ and $e$, or neither of them, or else the 4-cocircuit intersects the 4-circuit $\{a, b, d, e\}$ at a single element. But $\{f, g, h, i\}$ cannot be a 4 -cocircuit, so $\{d, e, h, i\}$ is a 4 -cocircuit. Let $A$ be a 4 -circuit containing $h$ and $a$. Suppose $b \in A$. Then $A$ must contain an additional element of the 4-cocircuit $G=\{b, c, e, g\}$. We can't have $c \in A$ or else $A$ contains the triangle $\{a, b, c\}$. We also cannot have $e$ or $g$ in $A$ or else $A$ contains a single element of the 4-cocircuit $S=\{d, e, f, g\}$. Therefore $b \notin A$. But $A$ must still contain an additional element of the 4 -cocircuit $R=\{a, b, d, f\}$. Hence one of $f$ and $d$ is in $A$. The fourth element of $A$ must be an additional element of the 4-cocircuit $S=\{d, e, f, g\}$, but $A$ cannot contain a single element of the 4-cocircuit $G=\{b, c, e, g\}$. Hence both $d$ and $f$ are members of $A$, so that $A=\{a, d, f, h\}$ is a 4 -circuit. Similarly a 4 -circuit containing $i$ and $a$ must be $\{a, d, f, i\}$. Therefore $r(\{a, d, f, h, i\})=3$. In the same way, $\{c, e, g, h\}$ and $\{c, e, g, i\}$ are 4-circuits, making $r(\{c, e, g, h, i\})=3$. But $\{a, d, f, h, i\}$ and $\{c, e, g, h, i\}$ complement the 4-cocircuits $R=\{a, b, d, f\}$ and $G=\{b, c, e, g\}$, making $\{a, d, f, h, i\}$ and $\{c, e, g, h, i\}$ hyperplanes. Therefore $r(M)=4$ and $r^{*}(M)=5$. The fact that $\{f, g, h, i\}$ is not a 4 -cocircuit means that $\{a, b, c, d, e\}$ is not a hyperplane. But $r(\{a, b, c, d, e\})=3$, so it must be properly contained in a hyperplane. We know that $f, g \notin \operatorname{cl}(\{a, b, c, d, e\})$, so one of $h$ and $i$ is on $\operatorname{cl}(\{a, b, c, d, e\})$. We cannot have both $h$ and $i$ in 
$\operatorname{cl}(\{a, b, c, d, e\})$, or else $\{f, g\}$ is a series pair, contradicting the 3-connectivity of $M$. Hence we may assume by relabelling that $h \in \operatorname{cl}(\{a, b, c, d, e\})$, making $\{a, b, c, d, e, h\}$ a hyperplane and $\{f, g, i\}$ a triad. But the triad $\{f, g, i\}$ and the 4-circuit $A=\{a, d, f, h\}$ share a single element, a contradiction.

Suppose $|E(M)|=10$. Then there are three additional elements $h, i, j \notin$ $\{a, b, c, d, e, f, g\}$. A 4-cocircuit containing $h$ and $i$ cannot contain any element of the triangle $\{a, b, c\}$, or else it would have to contain two other members of $\{a, b, c, d, e\}$. The 4-cocircuit must contain either both of $d$ and $e$, or neither of them, or else the 4-cocircuit intersects the 4-circuit $\{a, b, d, e\}$ at a single element. But $\{f, g, h, i\}$ cannot be a 4 -cocircuit, so $\{d, e, h, i\}$ is a 4-cocircuit. Similarly, 4-cocircuits containing $h$ and $j$, or $i$ and $j$, must contain both $d$ and $e$. Therefore $\{d, e, h, j\}$ and $\{d, e, i, j\}$ are also 4-cocircuits. A 4-circuit $A$ containing $b$ and $h$, must contain a member of $\{a, d, f\}$ and a member of $\{c, e, g\}$. But $A$ must also contain an additional element of the 4-cocircuit $\{d, e, h, i\}$, so either $d$ or $e$ is in $A$. Also, $A$ must contain an additional element of the 4 -cocircuit $\{d, e, i, j\}$, so both $d$ and $e$ are in $A$. Therefore $A=\{b, d, e, h\}$. Similarly, 4-circuits containing $b$ and $i$, and $b$ and $j$, must contain both $d$ and $e$. Therefore $\{b, d, e, i\}$ and $\{b, d, e, j\}$ are also 4 -circuit. But then $r(\{a, b, c, d, e, h, i, j\}=3$, so $\{f, g\}$ contains a cocircuit, contradicting the 3 -connectivity of $M$.

We can rule out matroids containing triangles and triads from our analysis. As we did for rank-2 flats in Lemma 3.3, we can restrict the size of a rank-3 flat by disallowing $U_{3,5}$-restrictions. A $U_{3,5}$-restriction is a rank35 -element set containing no loops, parallel pairs or triangles. Dually, a $U_{2,5}$-corestriction is a corank-3 5 -element set containing no coloops, series pairs or triads. We note that every 4 -element subset of a $U_{3,5}$-restriction is a 4-circuit and every 4-element subset of a $U_{2,5}$-corestriction is a 4-cocircuit. Conversely, the union of two 4-circuits that intersect at exactly 3 elements is a $U_{3,5}$-restriction, provided it contains no loops, parallel pairs or triangles, and the same dually for two 4-coircuits. The following results dualise to restrict the size of a corank-3 coflat by disallowing a $U_{2,5}$-corestriction.

Lemma 3.9. Let $M$ be a matroid with the 4-circuit-cocircuit property. If $|E(M)| \geq 7$, then $M$ cannot contain a $U_{3,6}$-restriction.

Proof. Let $\{a, b, c, d, e, f\}$ be a $U_{3,6}$-restriction in $M$ and $g \notin\{a, b, c, d, e, f\}$ be another element of $M$. Every 4-element subset of $\{a, b, c, d, e, f\}$ is a 
4-circuit. A 4-cocircuit containing $a$ and $g$ must contain 3 more elements from $\{a, b, c, d, e, f\}$ in order to not have a single element intersection with a 4-circuit, a contradiction.

Lemma 3.10. Let $M$ be a matroid with the 4-circuit-cocircuit property. If $|E(M)| \geq 8$ and $M$ contains no triangles or triads, then $M$ cannot contain a $U_{3,5}$-restriction.

Proof. By Lemma 3.9 and its dual, $r(M) \geq 4$ and $r^{*}(M) \geq 4$. Let $\{a, b, c, d, e\}$ be a $U_{3,5}$-restriction in $M$ and $x \notin\{a, b, c, d, e\}$ be another element of $M$. By Lemma 3.9, $x \notin \operatorname{cl}(\{a, b, c, d, e\})$. Every 4 element subset of $\{a, b, c, d, e\}$ is a 4-circuit. A 4-cocircuit containing $a$ and $x$ must contain two more elements from $\{a, b, c, d, e\}$ in order to not have a single element intersection with a 4 -circuit. By relabelling we can assume that $\{a, b, c, x\}$ is a 4-cocircuit. Suppose the 4-cocircuit containing $d$ and $x$ contains two from $\{a, b, c\}$, say $a$ and $b$ making $\{a, b, d, x\}$ a 4-cocircuit. Then $c, d \in \mathrm{cl}^{*}(\{a, b, x\})$ making $r^{*}(\{a, b, c, d, x\})=3$. The 4-cocircuit containing $e$ and $x$ must contain two elements from $\{a, b, c, d\}$, making $r^{*}(\{a, b, c, d, e, x\})=3$, in contradiction with the dual of Lemma 3.9. Therefore the 4-cocircuit containing $d$ and $x$ contains one element from $\{a, b, c\}$ and must contain $e$, so we may assume by relabelling that $\{a, d, e, x\}$ is a 4-cocircuit. By applying circuit elimination to the 4-cocircuits $\{a, b, c, x\}$ and $\{a, d, e, x\}$ there is a cocircuit contained in $\{a, b, c, d, e\}$. This means that $r^{*}(\{a, b, c, d, e\}) \leq 4$. But for all $x \notin\{a, b, c, d, e\}, x \in \mathrm{cl}^{*}(\{a, b, c, d, e\})$ so $r^{*}(M)=4$. Suppose $r(M)=4$. Then $|E(M)|=8$, and $\{a, b, c, d, e\}$ is a hyperplane. But this makes the complement of $\{a, b, c, d, e\}$ a triad, a contradiction. Therefore $r(M) \geq 5$ and $|E(M)| \geq 9$. This means that we can choose $y \notin \mathrm{cl}(\{a, b, c, d, e, x\})$. Any 4-set containing $x, y$ and two elements from $\{a, b, c, d, e\}$ must be independent, so the 4-circuit containing $x$ and $y$ must contain one and only one member of $\{a, b, c, d, e\}$. In order to avoid a single element intersection with either of the 4-cocircuits $\{a, b, c, x\}$ and $\{a, d, e, x\}$, this member must be $a$. Hence $\{a, x, y, z\}$ is a 4 -circuit for some element $z \notin\{a, b, c, d, e, x, y\}$. Let $w \notin\{a, b, c, d, e, x, y, z\}$ be the ninth element of $M$. The 4-cocircuit containing $a$ and $w$ must contain one additional member of $\{a, x, y, z\}$ and two additional members of $\{a, b, c, d, e\}$, a contradiction.

At this point we note that if $|E(M)|=8$ and $M$ has no triangles or triads, then $M$ is a rank-4 sparse paving matroid. For these matroids, every 
nonspanning circuit is a circuit-hyperplane. If $M$ is indeed a spike, this means that the choice of partition of $E(M)$ to get the legs of the spike is not necessarily unique. This makes these matroids particularly difficult to analyse. We will omit the full analysis of these matroids from this thesis

In Lemma 3.2, we showed that $M$ can't have a 5 -star without being a spike. We now extend that result to 4-stars, giving two families of matroids as non-spike exceptions. We need to define two more matroids. These matroids, along with the result of relaxing any combination of their circuithyperplanes, will be the only exceptions here. The 4-circuits are easy to see in the representations given. The fact that these matroids are self-dual gives us the 4-cocircuits. We will give a geometric representation for each of these matroids, and another for the matroids attained by relaxing all circuithyperplanes.

Let $M_{3}$ be the matroid represented over $\mathrm{GF}(2)$ by the following matrix.

$$
\left[\begin{array}{llllllllllll}
1 & 2 & 3 & 4 & 5 & 6 & 7 & 8 & 9 & 10 & 11 & 12 \\
0 & 1 & 0 & 1 & 0 & 0 & 0 & 0 & 0 & 0 & 1 & 1 \\
1 & 1 & 1 & 1 & 0 & 0 & 0 & 0 & 0 & 0 & 0 & 0 \\
0 & 0 & 1 & 1 & 0 & 1 & 0 & 1 & 0 & 0 & 0 & 0 \\
0 & 0 & 0 & 0 & 1 & 1 & 1 & 1 & 0 & 0 & 0 & 0 \\
0 & 0 & 0 & 0 & 0 & 0 & 1 & 1 & 0 & 1 & 0 & 1 \\
0 & 0 & 0 & 0 & 0 & 0 & 0 & 0 & 1 & 1 & 1 & 1
\end{array}\right]
$$

A geometric representation of $M_{3}$ is given in Figure 3.3. We note that $M_{3}$ contains 8 circuit-hyperplanes. These are the 6 -element sets containing either $\{1,4\}$ or $\{2,3\}$, either $\{5,8\}$ or $\{6,7\}$, and either $\{9,12\}$ or $\{10,11\}$. A geometric representation of the matroid $M_{3}^{-8}$, attained by relaxing all of these circuit-hyperplanes, is given in Figure 3.4.

Let $M_{4}$ be the matroid represented over GF(3) by the following matrix.

$$
\left[\begin{array}{cccccccccc}
1 & 2 & 3 & 4 & 5 & 6 & 7 & 8 & 9 & 10 \\
0 & 1 & 0 & 1 & 0 & 0 & 0 & 0 & 1 & 1 \\
1 & 1 & 1 & 1 & 0 & 0 & 0 & 0 & 0 & 0 \\
0 & 0 & 1 & 1 & 0 & 1 & 0 & 1 & 0 & 0 \\
0 & 0 & 0 & 0 & 1 & 1 & 1 & 1 & 0 & 0 \\
0 & 0 & 0 & 0 & 0 & 0 & 1 & 1 & 1 & -1
\end{array}\right]
$$




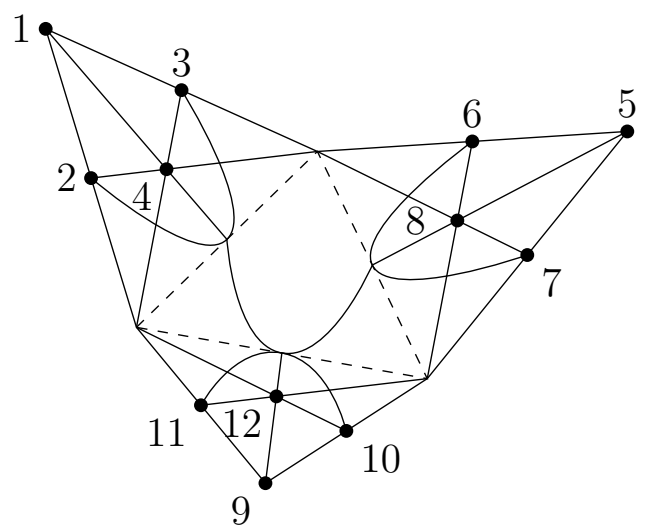

Figure 3.3: A geometric representation of $M_{3}$.

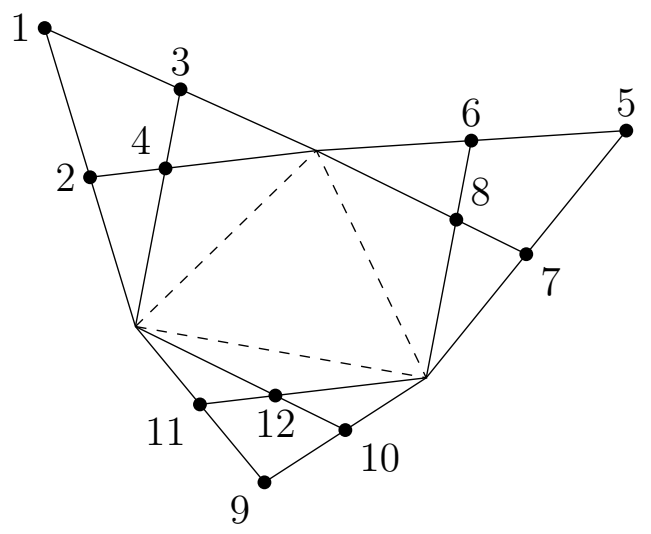

Figure 3.4: A geometric representation of $M_{3}^{-8}$.

A geometric representation of $M_{4}$ is given in Figure 3.5. We note that $M_{3}$ contains 4 circuit-hyperplanes. These are $\{1,4,5,8,9\},\{1,4,6,7,10\}$, $\{2,3,5,8,10\}$ and $\{2,3,6,7,9\}$. A geometric representation of the matroid $M_{4}^{-4}$, attained by relaxing all of these circuit-hyperplanes, is given in Figure 3.6.

We can show that there are matroids which are have all of the nonspanning circuits of $M_{4}^{-4}$, as well as either $\{1,4,5,8,9\}$ or $\{1,4,5,8,10\}$, either $\{1,4,6,7,9\}$ or $\{1,4,6,7,10\}$, either $\{2,3,5,8,9\}$ or $\{2,3,5,8,10\}$, and either $\{2,3,6,7,9\}$ or $\{2,3,6,7,10\}$, as circuit-hyperplanes. We call this class of matroids $T\left(M_{4}^{-4}\right)$.

Lemma 3.11. Let $M$ be a matroid with the 4-circuit-cocircuit property. If $|E(M)| \geq 9, M$ contains no triangles or triads, contains a 4-star and is not a spike, then it is isomorphic to either $M_{3}, M_{4}$, or one of their relaxations. 


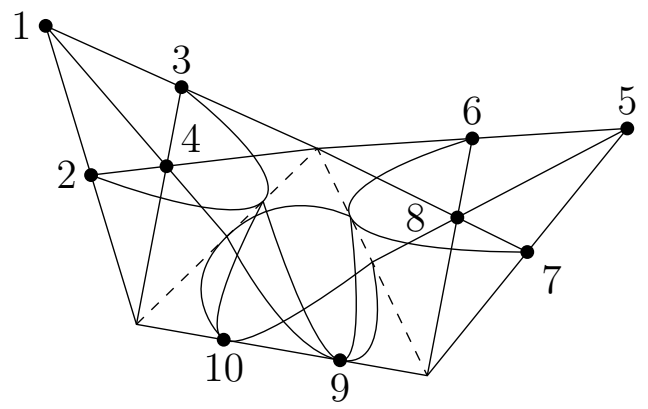

Figure 3.5: A geometric representation of $M_{4}$.

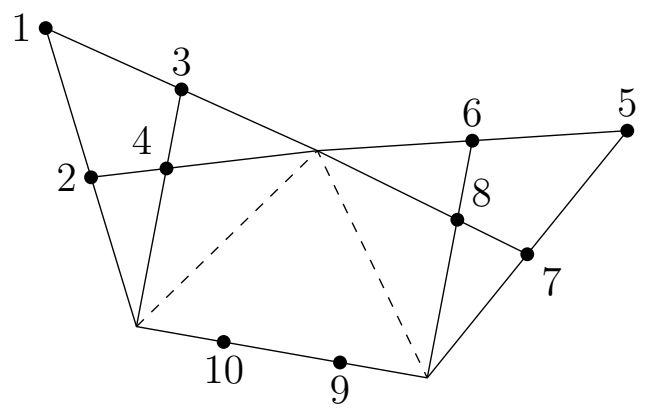

Figure 3.6: A geometric representation of $M_{4}^{-4}$.

Proof. Let $A$ be a 4-star in $M$. Let $A$ be labelled as in Definition 2.2 and let $x$ be an element not in $A$. For each arm $\left\{a_{i}, b_{i}\right\}$ of $A$, there is a 4 -cocircuit containing $x$ and $a_{i}$, which must contain $b_{i}$ or else it would have to contain an element from each of the other three arms of $A$, a contradiction. The fourth element cannot belong to $A$ or else the 4-cocircuit will intersect a circuit at a single element. Therefore the fourth element is an additional element $x_{i} \notin A \cup x$ and so $G_{1}=\left\{x, x_{1}, a_{1}, b_{1}\right\}, G_{2}=\left\{x, x_{2}, a_{2}, b_{2}\right\}, G_{3}=\left\{x, x_{3}, a_{3}, b_{3}\right\}$ and $G_{4}=\left\{x, x_{4}, a_{4}, b_{4}\right\}$ are 4 -cocircuits with the $x_{i}$ possibly not pairwise distinct.

Suppose $\left|\left\{x_{1}, x_{2}, x_{3}, x_{4}\right\}\right|=4$. Then the $x_{i}$ are pairwise distinct. Any 4-circuit containing $x$ must contain an element from each of the disjoint sets $\left\{x_{1}, a_{1}, b_{1}\right\},\left\{x_{2}, a_{2}, b_{2}\right\},\left\{x_{3}, a_{3}, b_{3}\right\}$ and $\left\{x_{4}, a_{4}, b_{4}\right\}$, a contradiction.

Suppose $\left|\left\{x_{1}, x_{2}, x_{3}, x_{4}\right\}\right|=3$. We may assume by relabelling that $x_{1}=x_{2}$ and $x_{1}, x_{3}$ and $x_{4}$ are pairwise disjoint. Let $C$ be some 4 -circuit containing $x$ and $a_{1}$. This 4-circuit must contain one from each of the disjoint sets $\left\{x_{1}, a_{2}, b_{2}\right\},\left\{x_{3}, a_{3}, b_{3}\right\}$ and $\left\{x_{4}, a_{4}, b_{4}\right\}$, a contradiction.

Suppose $\left|\left\{x_{1}, x_{2}, x_{3}, x_{4}\right\}\right|=2$. One case is that three of the $x_{i}$ are equal, 
so that by relabelling we may assume $x_{1}=x_{2}=x_{3} \neq x_{4}$. Let $C$ be some 4-circuit containing $x_{4}$ and $a_{1}$. Suppose $x \notin C$. Then $x_{1} \notin C$ or else $C$ must contain one from each of $\left\{a_{2}, b_{2}\right\},\left\{a_{3}, b_{3}\right\}$ and $\left\{a_{4}, b_{4}\right\}$. Therefore $b_{1} \in C$. The fourth element of $C$ must be one of $\left\{a_{4}, b_{4}\right\}$, so we may assume by relabelling that $a_{4} \in C$ and $C=\left\{a_{1}, b_{1}, a_{4}, x_{4}\right\}$. But then $\left\{a_{1}, b_{1}, a_{4}, b_{4}, x_{4}\right\}$ is a $U_{3,5}$-restriction, a contradiction. Hence $x \in C$. The fourth element of $C$ must belong to both $\left\{x_{1}, a_{2}, b_{2}\right\}$ and $\left\{x_{1}, a_{3}, b_{3}\right\}$, so must be $x_{1}$ making $C=\left\{a_{1}, x, x_{1}, x_{4}\right\}$ a 4-circuit. Similarly, a 4-circuit containing $x_{4}$ and $a_{2}$ must be $\left\{a_{2}, x, x_{1}, x_{4}\right\}$. But then $\left\{a_{1}, a_{2}, x, x_{1}, x_{4}\right\}$ is a $U_{3,5}$-restriction, a contradiction.

The other case where $\left|\left\{x_{1}, x_{2}, x_{3}, x_{4}\right\}\right|=2$ is that two of the $x_{i}$ are equal and so are the other two, so that by relabelling we may assume $x_{1}=x_{2} \neq$ $x_{3}=x_{4}$.

Claim 3.11.1. $M$ is isomorphic to $M_{3}$ or one of its relaxations.

Proof. Let $C$ be some 4-circuit containing $x$ and $a_{1}$. This 4-circuit must contain one from each of the sets $\left\{x_{1}, a_{2}, b_{2}\right\},\left\{x_{3}, a_{3}, b_{3}\right\}$ and $\left\{x_{3}, a_{4}, b_{4}\right\}$. The only way this is possible is to have $x_{3} \in C$.

Suppose for contradiction that $x_{1} \in C$, so that $C=\left\{x, x_{1}, x_{3}, a_{1}\right\}$. A 4-circuit containing $x$ and $b_{1}$ must similarly contain $x_{3}$ and an additional element from the 4 -cocircuit $G_{2}=\left\{x, x_{1}, a_{2}, b_{2}\right\}$. It cannot contain $x_{1}$ or else $\left\{x, x_{1}, x_{3}, a_{1}, b_{1}\right\}$ is a $U_{3,5}$-restriction. Hence we can assume by relabelling that it contains $b_{2}$ so that $\left\{x, x_{3}, b_{1}, b_{2}\right\}$ is a 4-circuit. A 4-circuit containing $x$ and $a_{2}$ must also contain $x_{3}$ and an additional element from the 4-cocircuit $G_{1}=\left\{x, x_{1}, a_{1}, b_{1}\right\}$. However this 4-circuit cannot contain $x_{1}$ or $a_{1}$ without overlapping $C=\left\{x, x_{1}, x_{3}, a_{1}\right\}$ at three elements creating a rank-5 3-set. It also cannot contain $b_{1}$ without overlapping $\left\{x, x_{3}, b_{1}, b_{2}\right\}$ at three elements creating a rank-5 3-set, a contradiction.

Therefore, the fourth element of $C$ cannot be $x_{1}$, so it is either $a_{2}$ or $b_{2}$. By relabelling we may assume $a_{2} \in C$ so that $C=\left\{x, x_{3}, a_{1}, a_{2}\right\}$. Similarly, a 4-circuit containing $x$ and $a_{3}$ must contain $x_{1}$ and either $a_{4}$ or $b_{4}$, so by relabelling we may assume that $\left\{x, x_{1}, a_{3}, a_{4}\right\}$ is a 4 -circuit. Also, a 4 -circuit containing $x$ and $b_{1}$ must contain $x_{3}$ and either $a_{2}$ or $b_{2}$. But if $\left\{x, x_{3}, b_{1}, a_{2}\right\}$ is a 4 -circuit then $\left\{x, x_{3}, a_{1}, a_{2}, b_{1}\right\}$ is a $U_{3,5}$-restriction contradicting Lemma 3.10. Hence $\left\{x, x_{3}, b_{1}, b_{2}\right\}$ is a 4 -circuit. Similarly $\left\{x, x_{1}, b_{3}, b_{4}\right\}$ is a 4 -circuit. 
Let $Q$ be some 4-cocircuit containing $a_{1}$ and $a_{3}$. If $b_{1} \in Q$ then the fourth element of $Q$ must belong to both of the 4-circuits $\left\{a_{2}, b_{2}, a_{3}, b_{3}\right\}$ and $\left\{a_{3}, b_{3}, a_{4}, b_{4}\right\}$, so $b_{3} \in Q$ making $Q=\left\{a_{1}, b_{1}, a_{3}, b_{3}\right\}$. But then $|C \cap Q|=$ 1 , a contradiction. Therefore $Q$ must contain one element from each arm of the 4-star $A$ and no other elements. In order to avoid single element intersections with the 4 -circuits $\left\{x, x_{3}, a_{1}, a_{2}\right\}$ and $\left\{x, x_{1}, a_{3}, a_{4}\right\}$ we must have $a_{3}, a_{4} \in Q$ making $Q=\left\{a_{1}, a_{2}, a_{3}, a_{4}\right\}$ a 4 -cocircuit. Similarly $\left\{a_{1}, a_{2}, b_{3}, b_{4}\right\}$, $\left\{b_{1}, b_{2}, a_{3}, a_{4}\right\}$ and $\left\{b_{1}, b_{2}, b_{3}, b_{4}\right\}$ must all be 4 -cocircuits.

Let $R$ be some 4-cocircuit containing $x_{1}$ and $x_{3}$. Then $R$ must contain an additional element from each of the 4 -circuits $\left\{x, x_{1}, a_{3}, a_{4}\right\},\left\{x, x_{1}, b_{3}, b_{4}\right\}$, $\left\{x, x_{3}, a_{1}, a_{2}\right\}$ and $\left\{x, x_{3}, b_{1}, b_{2}\right\}$. This is only possible if $x \in R$. In order to avoid intersecting one of the 4-circuits of the 4 -star at a single element, the fourth element of $R$ must be an additional element $y \notin A \cup\left\{x, x_{1}, x_{3}\right\}$, and so $R=\left\{x, y, x_{1}, x_{3}\right\}$ is a 4 -cocircuit.

Let $D$ be some 4-circuit containing $y$ and $a_{1}$. Then $D$ must contain an additional element from each of the 4-cocircuits $\left\{a_{1}, a_{2}, a_{3}, a_{4}\right\},\left\{a_{1}, a_{2}, b_{3}, b_{4}\right\}$ and $R=\left\{x, y, x_{1}, x_{3}\right\}$. This is only possible if $a_{2} \in D$. The fourth element must be one of $x, x_{1}$ and $x_{3}$. In order to avoid $D \cup C$ being a $U_{3,5}$-restriction, we must have $x_{1} \in D$ making $D=\left\{y, x_{1}, a_{1}, a_{2}\right\}$ a 4-circuit. Similarly, $\left\{y, x_{1}, b_{1}, b_{2}\right\},\left\{y, x_{3}, a_{3}, a_{4}\right\}$ and $\left\{y, x_{3}, b_{3}, b_{4}\right\}$ are 4-circuits.

Let $B$ be some 4-circuit containing $x$ and $y$. Then $B$ must contain an additional element from each of the 4-cocircuits $G_{1}=\left\{x, x_{1}, a_{1}, b_{1}\right\}, G_{2}=$ $\left\{x, x_{1}, a_{2}, b_{2}\right\}, G_{3}=\left\{x, x_{3}, a_{3}, b_{3}\right\}$ and $G_{4}=\left\{x, x_{3}, a_{4}, b_{4}\right\}$. This is only possible if $x_{1}, x_{3} \in B$ and so $B=R=\left\{x, y, x_{1}, x_{3}\right\}$. If $M$ contains some additional element $z \notin A \cup B$, then a 4-circuit containing $z$ and $x$ would similarly have to be $\left\{x, z, x_{1}, x_{3}\right\}$, creating a $U_{3,5}$-restriction, a contradiction. So $M$ contains no additional elements and $E(M)=A \cup B$. We now have the property that every pair of elements in $M$ belongs to a 4-circuit. We also now note that $\left\{a_{1}, a_{2}, b_{1}, b_{2}\right\} \cup B$ is a 4 -star with arms $\left\{a_{1}, a_{2}\right\},\left\{b_{1}, b_{2}\right\}$, $\left\{x, x_{3}\right\}$ and $\left\{y, x_{1}\right\}$, and $\left\{a_{3}, a_{4}, b_{3}, b_{4}\right\} \cup B$ is a 4 -star with arms $\left\{a_{3}, a_{4}\right\}$, $\left\{b_{3}, b_{4}\right\},\left\{x, x_{1}\right\}$ and $\left\{y, x_{3}\right\}$.

Let $S$ be a 4-cocircuit containing $y$ and $a_{1}$. Then $S$ cannot contain an element from each arm of the 4-star $A$, so $b_{1} \in S$. Also, $S$ cannot contain an element from each arm of the 4 -star $\left\{a_{3}, a_{4}, b_{3}, b_{4}\right\} \cup B$, so $x_{3} \in S$ and $S=\left\{y, x_{3}, a_{1}, b_{1}\right\}$ is a 4-cocircuit. Similarly, $\left\{y, x_{3}, a_{2}, b_{2}\right\},\left\{y, x_{1}, a_{3}, b_{3}\right\}$ and 
$\left\{y, x_{1}, a_{4}, b_{4}\right\}$ are 4-cocircuits.

Let $P$ be a 4-cocircuit containing $a_{1}$ and $b_{2}$. Then $P$ must contain an additional element from each of the 4 -circuits $\left\{a_{1}, b_{1}, a_{3}, b_{3}\right\},\left\{a_{1}, b_{1}, a_{4}, b_{4}\right\}$, $\left\{a_{1}, b_{2}, x, x_{3}\right\}$ and $\left\{a_{1}, a_{2}, y, x_{1}\right\}$. This is only possible if $b_{1}, a_{2} \in P$ so $P=$ $\left\{a_{1}, b_{1}, a_{2}, b_{2}\right\}$ is a 4 -cocircuit. Similarly $\left\{a_{3}, b_{3}, a_{4}, b_{4}\right\}$ is a 4 -cocircuit. We now have the property that every pair of elements in $M$ belongs to a 4cocircuit as well as a 4-circuit.

From here that we can easily check using circuit elimination that every 6 -element set we get by removing the common element from the union of two 4-circuits that overlap at exactly one element, is a 6-circuit. If $M$ contains no other non-spanning circuits, then by comparing non-spanning circuits, $M$ is isomorphic to $M_{3}^{-8}$.

We can easily check that $M$ can contain no other 4-circuits and no 5circuits. We can also check that other than the 6-circuits mentioned above, the only 6-circuits we can have are the eight circuit-hyperplanes in $M_{3}$. Hence $M$ is isomorphic to $M_{3}$ or one of its relaxations.

Now suppose $\left|\left\{x_{1}, x_{2}, x_{3}, x_{4}\right\}\right|=1$. This means that $x_{1}=x_{2}=x_{3}=x_{4}$.

Claim 3.11.2. The matroid $M$ is isomorphic to one of $T\left(M_{4}^{-4}\right)$ or a relaxation of one of these matroids.

Proof. Let $C$ be a 4 -circuit containing $x$ and $a_{1}$. $C$ must contain an additional element from each of the 4-cocircuits $G_{2}=\left\{x, x_{1}, a_{2}, b_{2}\right\}, G_{3}=\left\{x, x_{1}, a_{3}, b_{3}\right\}$ and $G_{4}=\left\{x, x_{1}, a_{4}, b_{4}\right\}$. This is only possible if $x_{1} \in C$.

Suppose for contradiction that there is some additional element $y \in C$ so that $C=\left\{x, x_{1}, a_{1}, y\right\}$. Then by Lemma 2.3, a 4-cocircuit containing $a_{1}$ and $a_{2}$ must contain either both $b_{1}$ and $b_{2}$, or one of each of $\left\{a_{3}, b_{3}\right\}$ and $\left\{a_{4}, b_{4}\right\}$. In either case, this 4 -cocircuit intersects $C$ at a single element, a contradiction.

Now suppose for contradiction that $b_{1} \in C$ so that $C=\left\{x, x_{1}, a_{1}, b_{1}\right\}$. As before, a 4-cocircuit containing $a_{1}$ and $a_{2}$ must contain either both $b_{1}$ and $b_{2}$, or one of each of $\left\{a_{3}, b_{3}\right\}$ and $\left\{a_{4}, b_{4}\right\}$. In order to avoid a single element intersection with $C$, the 4 -cocircuit has to be $\left\{a_{1}, b_{1}, a_{2}, b_{2}\right\}$. Similarly, $\left\{a_{1}, b_{1}, a_{3}, b_{3}\right\}$ and $\left\{a_{1}, b_{1}, a_{4}, b_{4}\right\}$ must be 4 -cocircuits. A 4 -circuit containing $x$ and $a_{2}$ must contain $x_{1}$. It cannot contain $a_{1}$ or $b_{1}$ without intersecting the 4-cocircuit $\left\{a_{1}, b_{1}, a_{3}, b_{3}\right\}$ at a single element. But it must contain an 
additional element from the 4 -cocircuit $\left\{a_{1}, b_{1}, a_{2}, b_{2}\right\}$. Hence $\left\{x, x_{1}, a_{2}, b_{2}\right\}$ is a 4-circuit. Similarly, $\left\{x, x_{1}, a_{3}, b_{3}\right\}$ and $\left\{x, x_{1}, a_{4}, b_{4}\right\}$ are 4-circuits. But that means that $A \cup\left\{x, x_{1}\right\}$ is a 5-star whose arms are the four arms of the 4-star $A$ and $\left\{x, x_{1}\right\}$. By Lemma 3.2, $M$ must be a spike, a contradiction.

We know $C$ contains $x, x_{1}$ and $a_{1}$ but cannot contain $b_{1}$ or a new element. Therefore the fourth element of $C$ must lie on one of the other three arms of the 4-star $A$. By relabelling we can assume that $a_{2} \in C$ so that $C=\left\{x, x_{1}, a_{1}, a_{2}\right\}$. Let $R$ be a 4 -cocircuit containing $a_{1}$ and $b_{2}$. By Lemma 2.3, $R$ must contain either both $b_{1}$ and $a_{2}$, or one of each of $\left\{a_{3}, b_{3}\right\}$ and $\left\{a_{4}, b_{4}\right\}$. But $R$ must also contain an additional element of $C=\left\{x, x_{1}, a_{1}, a_{2}\right\}$. Hence $a_{2}, b_{1} \in R$ so that $R=\left\{a_{1}, a_{2}, b_{1}, b_{2}\right\}$. Note that $R$ is also a 4 -circuit, so a quad. As with $C$, a 4 -circuit containing $x$ and $b_{1}$ must contain $x_{1}$ and an element on one of the other three arms of the 4-star $A$. But it must also contain an additional element of the quad $R=\left\{a_{1}, a_{2}, b_{1}, b_{2}\right\}$. Therefore the fourth element of this 4-circuit must be either $a_{2}$ or $b_{2}$. But it can't be $a_{2}$ or else $\left\{x, x_{1}, a_{2}, b_{1}, a_{1}\right\}$ is a $U_{3,5}$-restriction. Hence the fourth element is $b_{2}$ and $\left\{x, x_{1}, b_{1}, b_{2}\right\}$ is a 4 -circuit. As with $C$, a 4 -circuit containing $x$ and $a_{3}$ must contain $x_{1}$ and an element on one of the other three arms of the 4 -star $A$. But it cannot contain an element of the quad $R=\left\{a_{1}, a_{2}, b_{1}, b_{2}\right\}$. Therefore it must contain an element from the arm $\left\{a_{4}, b_{4}\right\}$. By relabelling we can assume it contains $a_{4}$, making $\left\{x, x_{1}, a_{3}, a_{4}\right\}$ a 4-circuit. Similarly, a 4-circuit containing $x$ and $b_{3}$ must contain $x_{1}$ and an element from the arm $\left\{a_{4}, b_{4}\right\}$. It cannot contain $a_{4}$ or else $\left\{x, x_{1}, b_{3}, a_{3}, a_{4}\right\}$ is a $U_{3,5}$-restriction. Hence $\left\{x, x_{1}, b_{3}, b_{4}\right\}$ is a 4 -circuit. We know $M$ cannot contain an additional element $y$ or else a 4-cocircuit containing $y$ and $a_{1}$ must contain $a_{2}$ (the other element on the arm $\left.\left\{a_{1}, a_{2}\right\}\right)$ and therefore must contain $x$ or $x_{1}$ in order to avoid intersecting the 4-circuits $C=\left\{x, x_{1}, a_{1}, a_{2}\right\}$ and $\left\{x, x_{1}, b_{1}, b_{2}\right\}$ at a single element, creating a single element intersection with the 4-circuit $\left\{x, x_{1}, a_{3}, a_{4}\right\}$. We now have that every pair of elements in $M$ belongs to a 4-circuit. Similarly to how we found the 4-cocircuit $R$, a 4-cocircuit containing $a_{3}$ and $b_{4}$ must be a quad $\left\{a_{3}, a_{4}, b_{3}, b_{4}\right\}$. A 4-cocircuit containing $a_{1}$ and $a_{3}$ must contain either both $b_{1}$ and $b_{3}$, or one of each of $\left\{a_{2}, b_{2}\right\}$ and $\left\{a_{4}, b_{4}\right\}$. It must also contain an additional element from each of the 4-circuits $C=\left\{x, x_{1}, a_{1}, a_{2}\right\}$ and $\left\{x, x_{1}, a_{3}, a_{4}\right\}$. Therefore $\left\{a_{1}, a_{2}, a_{3}, a_{4}\right\}$ is a 4 -cocircuit. Similarly, $\left\{a_{1}, a_{2}, b_{3}, b_{4}\right\},\left\{b_{1}, b_{2}, a_{3}, a_{4}\right\}$ and $\left\{b_{1}, b_{2}, b_{3}, b_{4}\right\}$ are 
4-cocircuits. Note that $A$ is now also a 4 -costar with arms $\left\{a_{1}, a_{2}\right\},\left\{b_{1}, b_{2}\right\}$, $\left\{a_{3}, a_{4}\right\}$ and $\left\{b_{3}, b_{4}\right\}$. We now have that every pair of elements in $M$ belongs to a 4-cocircuit as well as a 4-circuit.

Let the 4-circuit $C_{1}$ be either $\left\{x, x_{1}, a_{1}, a_{2}\right\}$ or $\left\{x, x_{1}, b_{1}, b_{2}\right\}$ and let the 4-circuit $C_{2}$ be either $\left\{x, x_{1}, a_{3}, a_{4}\right\}$ or $\left\{x, x_{1}, b_{3}, b_{4}\right\}$. We can easily check using circuit elimination that every 5 -element set we get by removing either $x$ or $x_{1}$ from the union of $C_{1}$ and $C_{2}$ is a 5 -circuit. This method gives us different 5 -circuits. If $M$ contains no other non-spanning circuits, then by comparing non-spanning circuits, $M$ is isomorphic to $M_{4}^{-4}$.

We can easily check that $M$ can contain no other 4 -circuits. We can also check that other than the 5-circuits mentioned above, the only 5 -circuits we can have contain either $\left\{a_{1}, b_{2}\right\}$ or $\left\{a_{2}, b_{1}\right\}$, either $\left\{a_{3}, b_{4}\right\}$ or $\left\{a_{3}, b_{4}\right\}$, and either $x$ or $x_{1}$. These sets are all circuit-hyperplanes. However we cannot have both $x$ and $x_{1}$ in the closure of a 4-element set containing either $\left\{a_{1}, b_{2}\right\}$ or $\left\{a_{2}, b_{1}\right\}$, and either $\left\{a_{3}, b_{4}\right\}$ or $\left\{a_{3}, b_{4}\right\}$, or else this set contains the whole matroid in its closure. We can check that there are no restrictions on whether a 5-circuit containing either $\left\{a_{1}, b_{2}\right\}$ or $\left\{a_{2}, b_{1}\right\}$, and either $\left\{a_{3}, b_{4}\right\}$ or $\left\{a_{4}, b_{3}\right\}$, contains $x$ or $x_{1}$. Hence $M$ is isomorphic to one of $T\left(M_{4}^{-4}\right)$ or a relaxation of one of these matroids.

This completes the proof of Lemma 3.11.

The remaining analysis deals with matroids containing no 4-stars or 4costars.

Lemma 3.12. Let $M$ be a matroid with the 4-circuit-cocircuit property. Suppose $|E(M)| \geq 9$, and $M$ contains no triangles or triads. Suppose $M$ has a 4-cocircuit $G$ with the property that no 4-circuit contains exactly three elements of $G$. Then every 4-circuit containing an element in $G$ and an element not in $G$ must contain two elements in $G$ and two elements not in $G$. Also, if distinct elements $x, y \notin G$ both belong to separate 4-circuits containing a pair $\{a, b\} \subseteq G$, say $\left\{x, x^{\prime}, a, b\right\}$ and $\left\{y, y^{\prime}, a, b\right\}$, then $x, x^{\prime}, y, y^{\prime}$ are pairwise distinct and $\left\{x, x^{\prime}, y, y^{\prime}\right\}$ is a 4-circuit making $\left\{a, b, x, x^{\prime}, y, y^{\prime}\right\}$ a 3-star with arms $\{a, b\},\left\{x, x^{\prime}\right\}$ and $\left\{y, y^{\prime}\right\}$. If there are any other elements not in $G$ contained in a 4-circuit containing $a$ and $b$, then they must also belong to a star containing this 3-star. 
Proof. Let $C$ be a 4 -circuit containing $x \notin G$ and $a \in G$. Then $|G \cap C| \neq 1,3$, so $C$ must contain exactly two elements in $G$ and two elements not in $G$. Now suppose $\left\{x, x^{\prime}, a, b\right\}$ and $\left\{y, y^{\prime}, a, b\right\}$ are distinct 4-circuits with $a, b \in G$ and $x, x^{\prime}, y, y^{\prime} \notin G$. Clearly $x \neq x^{\prime}$ and $y \neq y^{\prime}$. In order to avoid a $U_{3,5^{-}}$ restriction, we cannot have $\left|\left\{x, x^{\prime}, a, b\right\} \cap\left\{y, y^{\prime}, a, b\right\}\right|=3$. We also cannot have $\left|\left\{x, x^{\prime}, a, b\right\} \cap\left\{y, y^{\prime}, a, b\right\}\right|=4$ or else the 4 -circuits are not distinct. Therefore $\left|\left\{x, x^{\prime}, a, b\right\} \cap\left\{y, y^{\prime}, a, b\right\}\right|=2$, making $x, x^{\prime}, y, y^{\prime}$ pairwise distinct. By circuit elimination there is a circuit contained in $\left\{x, x^{\prime}, a, b\right\} \cup\left\{y, y^{\prime}, a, b\right\}-$ $b=\left\{x, x^{\prime}, y, y^{\prime}, a\right\}$. In order to avoid a single element intersection with the cocircuit $G, a$ cannot belong to this circuit. In order to avoid a triangle the circuit must be $\left\{x, x^{\prime}, y, y\right\}$ so that $\left\{a, b, x, x^{\prime}, y, y^{\prime}\right\}$ is a 3 -star with arms $\{a, b\},\left\{x, x^{\prime}\right\}$ and $\left\{y, y^{\prime}\right\}$. Inductively, for each additional element $z \notin G$ contained in 4-circuit containing $a$ and $b$, there must be an additional element $z^{\prime}$ so that the 4-circuit is $\left\{z, z^{\prime}, a, b\right\}$, and $\left\{z, z^{\prime}\right\}$ forms a 4-circuit with each of the other arms of the star, making $\left\{z, z^{\prime}\right\}$ an arm of a larger star.

Lemma 3.13. Let $M$ be a matroid with the 4-circuit-cocircuit property. Suppose $|E(M)| \geq 9, M$ contains no triangles or triads, and no 4-stars or 4costars. Let $G=\{a, b, c, d\}$ be a 4-cocircuit of $M$ with the property that no 4-circuit contains exactly three elements of $G$ and let $e \notin G$ be an additional element of $M$. Then, up to labels, either $e$ is contained in two 4-circuits containing complementary pairs $\{a, b\}$ and $\{c, d\}$, or $e$ is contained in three 4-circuits, each containing one of the three pairs in $G$ containing an element $a,\{a, b\},\{a, c\}$ and $\{a, d\}$.

Proof. Suppose $e$ does not belong to two 4-circuits containing complementary pairs of $G$. We may assume by relabelling that $\{a, b, e, f\}$ is a 4 -circuit containing $a$ and $e$, for an additional element $f \notin G \cup e$. There cannot be a 4-circuit containing $\{c, d\}$ and $e$. Therefore a 4-circuit containing $c$ and $e$ must contain one of $a$ and $b$, so we may assume by relabelling that $\{a, c, e, g\}$ is a 4 -circuit for some additional element $g \notin G \cup\{e, f\}(g \neq f$ otherwise we have a $U_{3,5}$-restriction). Now we know that there cannot be a 4 -circuit containing $\{b, d\}$ and $e$. Therefore a 4-circuit containing $d$ and $e$ must contain $a$ and some additional element $h \notin G \cup\{e, f\}(h \neq f, g$ otherwise we have a $U_{3,5}$-restriction) making $\{a, d, e, h\}$ a 4 -circuit.

One type of 4-cocircuit with this property is a quad. The following lemmas deal with matroids that contain a quad. 
Lemma 3.14. Let $M$ be a matroid with the 4-circuit-cocircuit property. Suppose $|E(M)| \geq 9, M$ contains no triangles or triads, and no 4-stars or 4costars. If $M$ has a quad $Q=\{a, b, c, d\}$, then up to labelling there cannot be an element $e \notin Q$ contained in three 4-circuits, each containing one of the three pairs in $G$ containing an element $a,\{a, b\},\{a, c\}$ and $\{a, d\}$.

Proof. Suppose there is such an element $e$. The fourth element in each of the three 4-circuits must be additional distinct elements in order to avoid a $U_{3,5}$-restriction. Hence $\{a, b, e, f\},\{a, c, e, g\}$ and $\{a, d, e, h\}$ are 4-circuits with $f, g, h \notin Q \cup e$ and distinct. Let $i$ be an additional element of $M$. Then the 4-cocircuit $G$ containing $a$ and $i$, must contain an additional element of the quad $Q$. By relabelling we may assume that $b \in G$. The fourth element cannot be $f$ in order to avoid a three element intersection with the 4-circuit $\{a, b, e, f\}$. But $G$ must contain an additional element of the other two 4-circuits $\{a, c, e, g\}$ and $\{a, d, e, h\}$, a contradiction.

Corollary 3.15. Let $M$ be a matroid with the 4-circuit-cocircuit property. Suppose $|E(M)| \geq 9, M$ contains no triangles or triads, and no 4-stars or 4-costars. If $M$ has a quad $Q=\{a, b, c, d\}$, then every element $x \notin Q$ is contained in two 4-circuits containing complementary pairs of elements of $Q$.

Proof. The result follows immediately from Lemmas 3.13 and 3.14, taking the quad $Q$ as the 4-cocircuit $G$.

Lemma 3.16. Let $M$ be a matroid with the 4-circuit-cocircuit property. Suppose $|E(M)| \geq 9, M$ contains no triangles or triads, and no 4-stars or 4costars. If $M$ has a quad $Q=\{a, b, c, d\}$, then there cannot be an element $e \notin Q$ contained in two 4-circuits, each containing one of the three pairs in $G$ containing an element $a$, say $\{a, b\}$ and $\{a, c\}$.

Proof. Suppose there is such an element $e$. The fourth element in each of the two 4-circuits must be additional distinct elements in order to avoid a $U_{3,5^{-}}$ restriction. Hence $\{a, b, e, f\}$ and $\{a, c, e, g\}$ are 4 -circuits with $f, g \notin Q \cup e$ and distinct. By Lemma 3.14, there is no 4-circuit containing $\{a, d\}$ and $e$. A 4-circuit containing $d$ and $e$ cannot contain $a$ so it must contain one of $b$ and $c$. Therefore we may assume by relabelling that $\{c, d, e, h\}$ is a 4 -circuit for some $h \notin Q \cup\{e, h\}$ but possibly $h=f$. 
Suppose $h \neq f$. We know that

$$
\begin{aligned}
\sqcap(Q, E(M)-Q) & =r(Q)+r(E(M)-Q)-r(M) \\
& =3+(r(M)-1)-r(M) \\
& =2 .
\end{aligned}
$$

Therefore $\sqcap(Q,\{e, f, g, h\}) \leq \sqcap(Q, E(M)-Q)=2$. Clearly,

$$
\begin{aligned}
r(Q \cup\{e, f, g, h\}) & =r(Q \cup e) \\
& =r(Q)+1 \\
& =4,
\end{aligned}
$$

as $e \notin \operatorname{cl}(Q)$ or else we would have a $U_{3,5}$-restriction. Hence

$$
\begin{array}{r}
r(Q)+r(\{e, f, g, h\})-r(Q \cup\{e, f, g, h\}) \leq 2 \\
3+r(\{e, f, g, h\})-4 \leq 2 \\
r(\{e, f, g, h\}) \leq 3 .
\end{array}
$$

So $\{e, f, g, h\}$ is a 4 -circuit. Let $i \notin Q \cup\{e, f, g, h\}$ be an additional element of $M$ and let $G$ be a 4-cocircuit containing $e$ and $i$. The two other elements of $G$ must be an additional element from each of the 4-circuits $\{a, b, e, f\}$ and $\{c, d, e, h\}$, exactly one of which must belong to the 4-circuit $\{a, c, e, g\}$. But $G$ must contain an additional element from the 4 -circuit $\{e, f, g, h\}$. Therefore we may assume by relabelling that $f \in G$. The fourth element of $G$ must be $c$ so that $G=\{c, e, f, i\}$. But then $G$ intersects with $Q$ at the single element $c$, a contradiction.

Therefore $h=f$. This makes $Q \cup\{e, f\}$ a 3 -star with arms $\{a, b\},\{c, d\}$ and $\{e, f\}$. Since $E(M) \geq 9$, there must be two additional elements $i, j \notin$ $Q \cup\{e, f, g\}$.

Let $G$ be a 4-cocircuit containing $g$ and $i$ and $R$ be a 4-cocircuit containing containing $g$ and $j$. Both of these 4-cocircuits must contain an additional element of the 4-circuit $\{a, c, e, g\}$. But $a, c$ and $e$ are all on an arm of the 3star $Q \cup\{e, f\}$. Since the 4-cocircuits can only contain one more element, they must contain the other element on that arm. Hence the other two elements of $G$ and $R$ must be one of the arms of the 3 -star. Both $G$ and $R$ must 
contain a different arm of the 3 -star or else their union is a $U_{2,5}$-corestriction. Therefore at least one of $G$ and $R$ must contain one of $\{a, b\}$ and $\{c, d\}$. By relabelling we can assume that $G=\{a, b, g, i\}$ is a 4 -cocircuit. There must be a cocircuit contained in $Q \cup G-b=\{a, c, d, g, i\}$. In order to avoid a single element intersection with the 4-circuit $\{a, b, e, f\}$, this cocircuit must be $\{c, d, g, i\}$, making $Q \cup\{g, i\}$ a 3-costar with arms $\{a, b\},\{c, d\}$ and $\{g, i\}$. If $R$ contains either $\{a, b\}$ or $\{c, d\}$, then we have a $U_{2,5}$-corestriction. Hence $R=\{e, f, g, j\}$ is a 4 -cocircuit.

Let $C$ be a 4 -circuit containing $a$ and $i$. Then $C$ must contain exactly one additional element from the quad $\{a, b, c, d\}$ in order to avoid intersecting it at a single element or forming a $U_{2,5}$-corestriction. Hence exactly one of $\{b, c, d\}$ is in $C$. If $b \in C$ then $C$ contains a single element of the 4-cocircuit $\{c, d, g, i\}$. So we must have $g \in C$. But then $C$ intersects $R=\{e, f, g, j\}$ at a single element. Therefore one of $c$ and $d$ is in $C$. The fourth element cannot be in $R=\{e, f, g, j\}$ or else $C$ would intersect it at a single element. Therefore the fourth element must be some additional element $k \notin Q \cup R \cup i$. But then the 4-cocircuit containing $g$ and $k$ must contain one of the arms of the 3 -star $Q \cup\{e, f\}$, creating a $U_{2,5}$-corestriction when we take the union with one of the 4-cocircuits $\{a, b, g, i\},\{c, d, g, i\}$ and $\{e, f, g, i\}$, a contradiction.

Corollary 3.17. Let $M$ be a matroid with the 4-circuit-cocircuit property. Suppose $|E(M)| \geq 9, M$ contains no triangles or triads, and no 4-stars or 4-costars. If $M$ has a quad $Q=\{a, b, c, d\}$, then every element $x \notin Q$ is contained in two 4-circuits containing complementary pairs of elements of $Q$, and no other 4-circuits containing elements of $Q$.

Proof. The result follows immediately from Corollary 3.15 and Lemma 3.16, since every other pair of elements of $Q$ apart from the complementary pairs, must be copunctual to those pairs.

Let $M_{5}$ be the matroid represented over GF(4) by the following matrix.

$$
\left[\begin{array}{cccccccccc}
1 & 2 & 3 & 4 & 5 & 6 & 7 & 8 & 9 & 10 \\
1 & 1 & 1 & 1 & 0 & 0 & 0 & 0 & 0 & 0 \\
0 & 1 & 0 & 1 & 0 & 0 & 0 & 1 & 1 & 0 \\
0 & 0 & 1 & 1 & 0 & 1 & 1 & 0 & 0 & 0 \\
0 & 0 & 0 & 0 & 0 & 0 & \omega & \omega & \omega^{2} & 1 \\
0 & 0 & 0 & 0 & 1 & 1 & 1 & 1 & 1 & \omega
\end{array}\right]
$$


A geometric representation of $M_{5}$ is given in Figure 3.7.

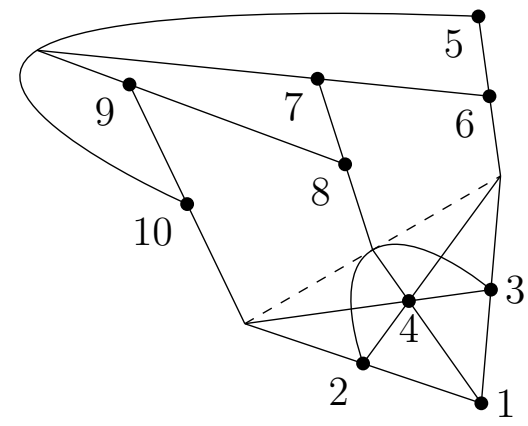

Figure 3.7: A geometric representation of $M_{5}$.

Lemma 3.18. Let $M$ be a matroid with the 4-circuit-cocircuit property. Suppose $|E(M)| \geq 9, M$ contains no triangles or triads, and no 4-stars or 4costars. If $M$ has a quad, then $M$ is isomorphic to $M_{5}$.

Proof. Let $Q=\{a, b, c, d\}$ be a quad in $M$. Let $e \notin Q$ be an additional element of $M$. By relabelling we can assume that $e$ belongs to 4-circuits $\{a, b, e, f\}$ and $\{c, d, e, g\}$, with $f, g \notin Q \cup e$ additional element of $M$ and possibly equal.

Suppose $g \neq f$. By Corollary 3.17, the only 4-circuit other than $\{a, b, e, f\}$ containing $f$ and an element of $Q$ must contain the pair $\{c, d\}$. Hence $\{c, d, f, h\}$ is a 4 -circuit for some $h \notin Q \cup\{e, f, g\}$, making $\{c, d, e, g, f, h\}$ a 3 -star with arms $\{c, d\},\{e, g\}$ and $\{f, h\}$. Similarly $\{a, b, g, i\}$ is a 4-circuit for some $i \notin Q \cup\{e, f, g\}$, making $\{a, b, e, f, g, i\}$ a 3 -star with arms $\{a, b\}$, $\{e, f\}$ and $\{g, i\}$. The 3 -stars mean that $\{e, f, g\}$ spans $h$ and $i$, so we must have $i=h$ in order to avoid a $U_{3,5}$-restriction, so $\{e, f, g, h\}$ is 4-circuit. Let $j \notin Q \cup\{e, f, g, h\}$ be an additional element of $M$. A 4-cocircuit $G$ containing $e$ and $i$ must contain an additional element from each of the 4-circuits $\{a, b, e, f\}$ and $\{c, d, e, g\}$. In order to avoid intersecting $\{e, f, g, h\}, G$ must contain exactly one of $f$ and $g$. But then the fourth element of $G$ must belong to the quad $Q$, creating an intersection of a single element, a contradiction.

Therefore $g=f$. This makes $Q \cup\{e, f\}$ a 3-star with arms $\{a, b\},\{c, d\}$ and $\{e, f\}$. Let $h \notin Q \cup\{e, f\}$ be an additional element of $M$. A 4-circuit containing $a$ and $h$ cannot contain $b$ or we would create a 4 -star. Therefore we can assume by relabelling that $\{a, c, h, i\}$ is a 4 -circuit for some additional element $i \notin Q \cup\{e, f, h\}$. There must also be a 4 -circuit containing $h$ and 
$\{b, d\}$. The fourth element of this 4-circuit must be $i$ or else we can relabel to get into the case where $g \neq f$. Hence $\{b, d, h, i\}$ is a 4-circuit, making $Q \cup\{h, i\}$ a 3-star with arms $\{a, c\},\{b, d\}$ and $\{h, i\}$.

Let $j \notin Q \cup\{e, f, h, i\}$ be an additional element of $M$. A 4-circuit containing $a$ and $j$ cannot contain $b$ or $c$ or we would create a 4 -star. Therefore $\{a, d, j, k\}$ is a 4 -circuit for some additional element $k \notin Q \cup\{e, f, h, i, j\}$. Also, $\{b, c, j, k\}$ must be a 4-circuit, making $Q \cup\{j, k\}$ a 3 -star with arms $\{a, d\},\{b, c\}$ and $\{j, k\}$. There can be no other elements in $M$ without creating a 4-star.

A 4-cocircuit $G$ containing $a$ and $e$ cannot be the 4-circuit $\{a, b, e, f\}$ or else it would intersect the 4-circuit $\{a, c, h, i\}$ at a single element. Because $Q \cup\{e, f\}$ is a 3 -star with arms $\{a, b\},\{c, d\}$ and $\{e, f\}, G$ must contain one of $c$ and $d$. We may assume by relabelling that $c \in G$. The fourth element of $G$ must be an additional element of the 4-circuit $\{b, c, j, k\}$. We can't have $b \in G$ or else $G$ intersects the quad $Q$ at three elements. Therefore we may assume by relabelling that $G=\{a, c, e, k\}$ is a 4 -cocircuit. There must be a cocircuit contained in $Q \cup G-c=\{a, b, d, e, k\}$. But in order to avoid a single element intersection with the 4-circuit $\{a, c, h, i\}, a$ cannot be in the cocircuit so $\{b, d, e, k\}$ is a 4 -cocircuit since there can be no triads. This makes $Q \cup\{e, k\}$ a 3 -costar with arms $\{a, c\},\{b, d\}$ and $\{e, k\}$.

A 4 -cocircuit $R$ containing $a$ and $h$ cannot be the 4-circuit $\{a, c, h, i\}$ or else it would intersect the 4 -circuit $\{a, b, e, f\}$ at a single element. Because $Q \cup\{h, i\}$ is a 3 -star with arms $\{a, c\},\{b, d\}$ and $\{h, i\}, R$ must contain one of $b$ and $d$. We may assume by relabelling that $d \in R$. The fourth element of $R$ must be an additional element of the 4-circuit $\{a, b, e, f\}$. We can't have $b \in R$ or else $R$ intersects the quad $Q$ at three elements. By the dual of Corollary 3.17, e cannot belong to any other 4-cocircuits containing elements of $Q$. Hence $f \in R$ and $R=\{a, d, f, h\}$.

There must be a cocircuit contained in $Q \cup R-d=\{a, b, c, f, h\}$. But in order to avoid a single element intersection with the 4 -circuit $\{a, d, j, k\}, a$ cannot be in the cocircuit so $\{b, c, f, h\}$ is a 4-cocircuit since there can be no triads. This makes $Q \cup\{f, h\}$ a 3 -costar with arms $\{a, d\},\{b, c\}$ and $\{f, h\}$. A 4-cocircuit containing $j$ and elements of the quad $Q$ cannot contain the pairs $\{a, c\},\{b, d\},\{a, d\}$ or $\{b, c\}$ or else we would create a 4-costar. Hence there must be 4 -cocircuits containing $\{a, b, j\}$ and $\{c, d, j\}$. By the dual of 
corollary 3.17, these 4-cocircuits cannot contain any of $e, f, h$ or $k$. Therefore $\{a, b, i, j\}$ and $\{c, d, i, j\}$ must be 4-cocircuit, making $Q \cup\{i, j\}$ a 3-costar with arms $\{a, b\},\{c, d\}$ and $\{i, j\}$.

Let $C$ be a 4-circuit containing $e$ and $h$. Then $C$ must contain an additional element from each of the 4-cocircuits $\{a, c, e, k\}$ and $\{a, d, f, h\}$ But by Corollary 3.17, 4-circuits containing $e$ and elements of $Q$ must contain either $\{a, b\}$ or $\{c, d\}$, and 4-circuits containing $h$ and elements of $Q$ must contain either $\{a, c\}$ or $\{b, d\}$. Hence $C$ cannot contain elements of $Q$, so must contain $k$ and $f$, making $C=\{e, f, h, k\}$ a 4-circuit. Similarly, $\{e, i, j, k\}$ and $\{f, h, i, j\}$ must also be 4 -circuits, making $\{e, f, h, i, j, k\}$ a 4 -star with arms $\{e, k\},\{f, h\}$ and $\{i, j\}$. By the dual process, $\{e, f, h, i, j, k\}$ is also a 3 -costar with arms $\{e, f\},\{h, i\}$ and $\{j, k\}$.

From here it is straightforward to check that $M$ cannot have any more 4-circuits or 4-cocircuits than the ones already identified. There are also 12 5-circuits which must also be in $M$ due to circuit elimination. Again it is straightforward to check that $M$ cannot have any more 5-circuits. Given that $r(M)=5$, by comparing non-spanning circuits we can now see that $M \cong M_{5}$.

We now only have to consider matroids which don't contain a quad. The next lemma deals with matroids that contain a 4-circuit and a 4-cocircuit that overlap at exactly three elements.

Lemma 3.19. Let $M$ be a matroid with the 4-circuit-cocircuit property. If $|E(M)| \geq 9$, and $M$ contains no triangles, triads, or quads, then $M$ contains no 4-circuit $C$ and 4-cocircuit $R$ such that $|C \cap R|=3$.

Proof. Suppose for contradiction that $R=\{a, b, c, d\}$ is a 4-cocircuit of $M$ and $C=\{a, b, c, e\}$ is a 4 -circuit of $M$. Let $G$ be a 4 -cocircuit containing $d$ and $e$. Then $G$ must contain an additional element of the 4-circuit $C=$ $\{a, b, c, e\}$, so we may assume by relabelling that $c \in G$. Neither $a$ nor $b$ can be in $G$ or else $\{a, b, c, d, e\}$ is a $U_{2,5}$-corestriction. Hence $f \in G$ for some additional element $f \notin\{a, b, c, d, e\}$, so that $G=\{c, d, e, f\}$.

Claim 3.19.1. The set $\{a, b, c, d, e, f\}$ is spanning.

Proof. Suppose there is some element $g$ such that $g \notin \operatorname{cl}(\{a, b, c, d, e, f\})$. Let $D$ be a 4 -circuit containing $g$ and $c$. Then $D$ must contain an additional 
element $h \notin \operatorname{cl}(\{a, b, c, d, e, f\})$. The fourth element of $D$ must be an additional element of both of the 4-cocircuits $R=\{a, b, c, d\}$ and $G=\{c, d, e, f\}$. Therefore $d \in D$ so that $D=\{c, d, g, h\}$ is a 4 -circuit. By cocircuit elimination, there must be a cocircuit contained in $R \cup G-c=\{a, b, d, e, f\}$. In order to avoid a single element intersection with the 4-circuit $D=\{c, d, g, h\}, d$ cannot be a member of this cocircuit. Hence $\{a, b, e, f\}$ must be a 4-cocircuit, making $\{a, b, c, d, e, f\}$ a 3-costar with arms $\{a, b\},\{c, d\}$ and $\{e, f\}$.

Let $A$ be a 4-circuit containing $g$ and $a$. Then $A$ must contain some other element $x \notin \operatorname{cl}(\{a, b, c, d, e, f\})$. The fourth element of $A$ must belong to the same arm as $a$ in the 3 -costar $\{a, b, c, d, e, f\}$. Therefore $d \in A$ so that $A=$ $\{a, b, g, x\}$ is a 4 -circuit. Suppose $x=h$. Then by circuit elimination there is a circuit contained in $A \cup D-h=\{a, b, c, d, g\}$. But $g \notin \operatorname{cl}(\{a, b, c, d, e, f\})$, so $\{a, b, c, d\}$ is circuit. This makes $R=\{a, b, c, d\}$ a quad. Therefore $x \neq h$, so $x$ must be some additional element $i \notin\{a, b, c, d, e, f, g, h\}$. Hence $A=$ $\{a, b, g, i\}$. Similarly, a 4-circuit containing $g$ and $e$ must contain $f$, and an $x \notin \operatorname{cl}(\{a, b, c, d, e, f\})$ which isn't $h$ or $i$. Therefore $\{e, f, g, j\}$ is a 4-circuit for some additional element $j \notin\{a, b, c, d, e, f, g, h, i\}$.

Let $B_{1}$ be a 4-circuit containing $h$ and $a$ and let $B_{2}$ be a 4-circuit containing $j$ and $a$. Both $B_{1}$ and $B_{2}$ must contain some other element $x \notin$ $\operatorname{cl}(\{a, b, c, d, e, f\})$, and $b$. In either 4-circuit, $x$ cannot be $g$ or $i$ or else $\{a, b, g, h, i\}$ is a $U_{3,5}$-restriction. Therefore either $B_{1}=B_{2}=\{a, b, h, j\}$, or $B_{1}=\{a, b, h, k\}$ and $B_{2}=\{a, b, j, l\}$ for additional elements $k, l \notin$ $\{a, b, c, d, e, f, g, h, i, j\}$. Note that $k \neq l$ or else $\{a, b, h, j, k\}$ is a $U_{3,5^{-}}$ restriction. In the second case, by circuit elimination $\{g, i, h, k, b\},\{g, i, j, l, b\}$ and $\{h, k, j, l, b\}$ must all contain circuits. But in order to avoid a single element intersection with the 4-cocircuit $R=\{a, b, c, d\}$, we must have $\{g, i, h, k\},\{g, i, j, l\}$ and $\{h, k, j, l\}$ all 4 -circuits. This makes $\{a, b, g, h, i, j, k, l\}$ a 4-star with arms $\{a, b\},\{g, i\},\{h, k\}$ and $\{j, l\}$. Therefore $B_{1}=B_{2}=$ $\{a, b, h, j\}$ is a 4 -circuit. Similarly $\{c, d, i, j\}$ and $\{e, f, h, i\}$ are 4 -circuits. By circuit elimination, there is a circuit contained in $A \cup B_{1}-a=\{g, i, h, j, b\}$. In order to avoid a single element intersection with the 4-cocircuit $R=$ $\{a, b, c, d\},\{g, i, h, j\}$ must be a 4 -circuit. Continuing like this, we can show that $\{g, i, h, j\}$ must be a quad, a contradiction.

Therefore $\{a, b, c, d, e, f\}$ is a spanning set of the matroid $M$. Suppose $f \in$ $\operatorname{cl}(\{a, b, c, d, e\})$. Then $r(\{a, b, c, d, e, f\})=r^{*}(\{a, b, c, d, e, f\})=4$. Hence 
$\lambda(\{a, b, c, d, e, f\})=2$. Let $A$ be a subset of $M$ disjoint from $\{a, b, c, d, e, f\}$ and containing 3 elements. By the 3 -connectivity of $M, \lambda(A) \geq 2$. But

$$
\begin{aligned}
\lambda(A)= & \lambda(E(M)-A) \\
= & r(E(M)-A)+r^{*}(E(M)-A)-|E(M)-A| \\
\leq & r(E(M)-A)+\left(r^{*}(\{a, b, c, d, e, f\})+|E(M)-A-\{a, b, c, d, e, f\}|\right) \\
& -|E(M)-A| \\
= & 4+(4+|E(M)-A|-6)-|E(M)-A| \\
= & 2 \\
\leq & \lambda(A) .
\end{aligned}
$$

Hence $\lambda(A)=2$. But then $A$ is a triangle or a triad, a contradiction. Therefore $f \notin \mathrm{cl}(\{a, b, c, d, e\})$.

Let $A$ be a 4-circuit containing $d$ and $e$. Then $A$ must contain some element of $\{a, b, c\}$ and an additional element $g \in \operatorname{cl}(\{a, b, c, d, e\})$. By the duals of the previous two paragraphs, $\{a, b, c, d, e, g\}$ is a cospanning set of the matroid $M$, and $g \notin \mathrm{cl}^{*}(\{a, b, c, d, e\})$. We know now that $r(M)=$ $r(\{a, b, c, d, e, g\})=5$ and $r^{*}(M)=r^{*}(\{a, b, c, d, e, g\})=5$. This means that $M$ has 10 elements. From here, a finite case check will give us a contradiction.

Now Lemma 3.13 becomes useful again as any 4-cocircuit that we choose has the property that a 4-circuit cannot contain exactly three elements of it.

Lemma 3.20. Let $M$ be a matroid with the 4-circuit-cocircuit property. Suppose $|E(M)| \geq 9, M$ contains no triangles or triads, no 4-stars or 4-costars, no quads and no 4-circuit $C$ and 4-cocircuit $R$ such that $|C \cap R|=3$. Let $R=\{a, b, c, d\}$ be a 4-cocircuit of $M$. Then there cannot be an element $e \notin R$ contained in three 4-circuits, each containing a different pair of $R$ containing a single element $a$.

Proof. Suppose there is such an element $e$. The fourth element in each of the three 4-circuits must be additional distinct elements in order to avoid a $U_{3,5}$-restriction. Hence $\{a, b, e, f\},\{a, c, e, g\}$ and $\{a, d, e, h\}$ are 4-circuits with $f, g, h \notin R \cup e$ and distinct.

Claim 3.20.1. The set $\{a, f, g, h\}$ is a 4-cocircuit. 
Proof. Suppose $\{a, f, g, h\}$ is not a 4 -cocircuit. Let $G$ be a 4 -cocircuit containing $a$ and $f$. Then $G$ already contains two elements of the 4-circuit $\{a, b, e, f\}$. Hence $b, e \notin G$. But $G$ must contain an additional element each of the 4-circuits $\{a, c, e, g\}$ and $\{a, d, e, h\}$. Hence one of $c$ and $g$, and one of $d$ and $h$ are members of $G$. We can't have both $g, h \in G$ as $\{a, f, g, h\}$ is not a 4-cocircuit. Also we can't have both $c, d \in G$ or else $\{a, b, c, d, e\}$ is a $U_{2,5}$-corestriction. So by relabelling we can assume that $g, d \in G$. Hence $G=\{a, d, f, h\}$ is a 4 -cocircuit. We can assume by relabelling that a 4 cocircuit $a$ and $h$ is $\{a, b, g, h\}$. By cocircuit elimination there is a cocircuit contained in $G \cup\{a, b, g, h\}-a=\{b, d, f, g, h\}$. In order to avoid a single element intersection with the 4-circuit $\{a, c, e, g\},\{b, d, f, h\}$ must be a 4-cocircuit. Similarly, there is a cocircuit contained in $\{b, c, d, g, h\}$ which must be $\{c, d, g, h\}$, and a cocircuit contained in $\{b, c, d, f, g\}$ which must be $\{b, c, f, g\}$

Let $C$ be a 4 -circuit containing $b$ and $h$. Then $C$ cannot contain any additional elements from the 4 -cocircuits $\{b, d, f, h\}$ and $\{a, b, g, h\}$. Hence $a, d, f, g \notin C$. But $C$ must contain an additional element from the 4-cocircuit $\{c, d, g, h\}$. Therefore $c \in C$. Let $S$ be a 4 -cocircuit containing $b$ and $e$. Then $S$ cannot contain $a$ or $f$ from the 4 -circuit $\{a, b, f, e\}$. But $S$ must contain an additional element from each of the 4 -circuits $\{a, c, e, g\}$ and $\{a, d, e, h\}$. Therefore $S$ must contain one of $c$ and $g$, and one of $d$ and $h$. We cannot have both $c$ and $d$ in $S$ or else $\{a, b, c, d, e\}$ is a $U_{2,5}$-corestriction. We cannot have both $g$ and $h$ in $S$ or else $\{a, b, e, g, h\}$ is a $U_{2,5}$-corestriction. We cannot have both $c$ and $h$ in $S$ or else $|C \cap S| \geq 3$. Hence $d, g \in S$ and so $S=\{b, d, e, g\}$ is a 4-cocircuit. The 4-circuit $C$ cannot contain any more elements of the 4cocircuit $\{c, d, g, h\}$, so $d, g \notin C$. But $C$ must contain an additional element of the 4-cocircuit $S=\{b, d, e, g\}$. Therefore $e \in C$ and so $C=\{b, c, e, h\}$ is a 4-circuit. Similarly to how we found $C$, a 4-circuit containing $d$ and $f$ must contain $c$ and $e$. Therefore $\{c, d, e, f\}$ is a 4-circuit.

We now have three 4-circuits containing $c$ and $e$. These are $\{a, c, e, g\}$, $\{b, c, e, h\}$ and $\{c, d, e, f\}$. Therefore a 4-cocircuit containing $c$ and $e$ cannot contain any of $a, b, d, f, g$ and $h$. This contradicts the fact that this 4cocircuit must also contain an additional element of the 4 -circuit $\{a, d, f, e\}$.

Therefore $\{a, f, g, h\}$ is a 4 -cocircuit. A 4-cocircuit containing $e$ and one 
of $\{b, c, d, f, g, h\}$, cannot contain $a$ without intersecting one of the 4-circuits at three or four elements. Hence such a 4-cocircuit must contain one from each of $\{b, f\},\{c, g\}$ and $\{d, h\}$ in order to avoid intersecting one of the 4circuits at a single element. However such a 4-cocircuit cannot contain all of $\{b, c, d\}$ or $\{f, g, h\}$ or else it would intersect one of the other two 4-cocircuits at three elements. Therefore we may assume by relabelling that $\{b, d, e, g\}$ is a 4-cocircuit.

Claim 3.20.2. The set $\{c, e, f, h\}$ must also be a 4-cocircuit.

Proof. Suppose it is not. We know a 4-cocircuit containing $e$ and $f$ cannot contain both $g$ and $h$. It also cannot contain both $g$ and $d$ without forming a $U_{2,5}$-corestriction with the 4 -cocircuit $\{b, d, e, g\}$. By our assumption it cannot contain both $c$ and $h$. That only leaves $c$ and $d$ as a possibility, making $\{c, d, e, f\}$ a 4 -cocircuit. Similarly a 4-cocircuit containing $e$ and $h$ must be $\{b, c, e, h\}$. By cocircuit elimination on these two 4-cocircuits, there is a cocircuit contained in $\{b, c, d, f, h\}$. In order to avoid a single element intersection with the 4 -circuit $\{a, c, e, g\}$, this cocircuit must be $\{b, d, f, h\}$. Let $C$ be a 4 -circuit containing $b$ and $h$. In order to avoid intersecting the 4 -cocircuits $\{b, d, f, h\}$ and $\{b, c, e, h\}$ at three or four elements, we must have $d, f, c, e \notin C$. In order to avoid a single element intersection with either of the 4-cocircuits $\{a, b, c, d\}$ and $\{b, d, e, g\}$, we must have both $a, g \in C$ so that $C=\{a, b, g, h\}$. But then $C$ intersects the 4-cocircuit $\{a, f, g, h\}$ at three elements, a contradiction.

We now know that $\{c, e, f, h\}$ is a 4 -cocircuit. Let $G$ be a 4 -cocircuit containing $b$ and $h$. If $a \in G$, then we cannot have $e \in G$ or else $G$ shares three elements with the 4-circuit $\{a, b, e, f\}$. Also if $a \in G$, we cannot have either of $c$ or $g$ in $G$ without forming a $U_{2,5}$-corestriction with one of the 4-cocircuits $\{a, b, c, d\}$ or $\{a, f, g, h\}$. So in order to avoid a single element intersection with the 4-circuit $\{a, c, e, g\}$ we must have $a \notin G$. Similarly, $e \notin G$. Hence in order to avoid single element intersections with the 4-circuits $\{a, b, e, f\}$ and $\{a, d, e, g\}$, we must have $G=\{b, d, f, h\}$ a 4 -cocircuit. Let $C$ be a 4 -circuit containing $b$ and $h$. In order to avoid a three element intersection, we must have $d, f \notin C$. But then in order to avoid a single element intersection with one of the 4-cocircuits $\{a, b, c, d\},\{a, f, g, h\},\{b, d, e, g\}$ and $\{c, e, f, h\}, C$ must contain one from each of $\{a, c\},\{a, g\},\{e, g\}$ and $\{c, e\}$. But if both 
$a, e \in C$, then $C$ forms a $U_{3,5}$-restriction with the 4-circuit $\{a, b, e, f\}$. Hence $c, g \in C$ so that $C=\{b, c, g, h\}$ is a 4-circuit. Similarly, a 4-circuit containing $d$ and $f$ must be $\{c, d, f, g\}$. By circuit elimination, there must be a circuit contained in each of $\{b, d, f, h, c\}$ and $\{b, d, f, h, g\}$. However deleting any element from either of these 5-element sets gives a set intersecting the 4cocircuit $\{b, d, f, h\}$ at three or four elements. Hence both of these 5-element sets themselves must be circuits. A 4-cocircuit containing $c$ and $g$ must contain an additional element from each of these 5-element sets. But that creates a three element intersection with one of the 4 -circuits $\{b, c, g, h\}$ or $\{c, d, f, g\}$, a contradiction.

Corollary 3.21. Let $M$ be a matroid with the 4-circuit-cocircuit property. Suppose $|E(M)| \geq 9, M$ contains no triangles or triads, no 4-stars or 4costars, no quads and no 4-circuit $C$ and 4-cocircuit $R$ such that $|C \cap R|=3$. Let $R=\{a, b, c, d\}$ be a 4-cocircuit of $M$. Then every element $x \notin R$ is contained in two 4-circuits containing complementary pairs of elements of $Q$.

Proof. The result follows immediately from Lemmas 3.13 and 3.20, taking $R$ as the 4-cocircuit $G$.

Lemma 3.22. Let $M$ be a matroid with the 4-circuit-cocircuit property. Suppose $|E(M)| \geq 9, M$ contains no triangles or triads, no 4-stars or 4-costars, no quads and no 4-circuit $C$ and 4-cocircuit $R$ such that $|C \cap R|=3$. Let $R=\{a, b, c, d\}$ be a 4-cocircuit of $M$. Then there cannot be an element $e \notin R$ contained in two 4-circuits, each containing a different pair of $R$ containing a single element $a$, say $\{a, b\}$ and $\{a, c\}$.

Proof. This proof is basic case checking and is left to the reader.

Corollary 3.23. Let $M$ be a matroid with the 4-circuit-cocircuit property. Suppose $|E(M)| \geq 9, M$ contains no triangles or triads, no 4-stars or 4costars, no quads and no 4-circuit $C$ and 4-cocircuit $R$ such that $|C \cap R|=3$. Let $R=\{a, b, c, d\}$ be a 4-cocircuit of $M$. Then every element $x \notin R$ is contained in two 4-circuits containing complementary pairs of elements of $R$, and no other 4-circuits containing elements of $R$.

Proof. The result follows immediately from Corollary 3.21 and Lemma 3.22, since every other pair of elements of $R$ apart from the complementary pairs, contains exactly one element from each of those pairs. 
Lemma 3.24. Suppose $M$ is a matroid such that $|E(M)| \geq 9$, $M$ contains no triangles or triads, no 4-stars or 4-costars, no quads and no 4-circuit $C$ and 4-cocircuit $R$ such that $|C \cap R|=3$. Then $M$ contains a pair which is not contained in both a 4-circuit and a 4-cocircuit.

Proof. Let $R=\{a, b, c, d\}$ be a 4 -cocircuit. We now know that every element $x \in R$ is contained in two 4-circuits containing complementary pairs of elements of $R$, and no other 4-circuits containing elements of $R$. Again, the rest is easy case checking and is left to the reader.

This completes the analysis. We are now ready to state the main theorem in full. It follows easily from the propositions throughout this chapter.

Theorem 3.25. Let $M$ be a matroid in which every pair of elements belongs to both a 4-circuit and a 4-cocircuit.

- If $|E(M)| \geq 13$, then $M$ is a spike.

- If $|E(M)|=12$, then $M$ is a 6-spike, or is isomorphic to $M_{3}$, or a matroid attained by relaxing some of its circuit-hyperplanes.

- $|E(M)| \neq 11$.

- If $|E(M)|=10$, then $M$ is a 5-spike, or is isomorphic to one of $T\left(M_{4}^{-4}\right)$ or a relaxation of one of these matroids, or $M_{5}$.

- If $|E(M)|=9$, then $M$ is isomorphic to $M\left(K_{3,3}\right)$ or $M^{*}\left(K_{3,3}\right)$.

- If $|E(M)|=8$, then $M$ is a rank-4 sparse paving matroid, or is isomorphic to $M_{1}$ or $M_{2}$.

- If $|E(M)|=7$, then $M$ is isomorphic to $F_{7}, F_{7}^{-}, P_{7}, F_{7}^{*},\left(F_{7}^{-}\right)^{*}$ or $P_{7}^{*}$.

- If $|E(M)|=6$, then $M$ is a 3-spike.

- If $|E(M)| \leq 5$, then $|E(M)|=0,1$. 


\section{Bibliography}

[1] Geelen, J., Gerards, A., And Kapoor, A. The excluded minors for GF(4)-representable matroids. Journal of Combinatorial Theory, Series B 79, 2 (2000), 247-299.

[2] Oxley, J. Matroid Theory, second ed. Oxford Graduate Texts in Mathematics. Oxford University Press, New York, 2011.

[3] Oxley, J., And Whittle, G. A note on the non-spanning circuits of a matroid. European Journal of Combinatorics 12, 3 (1991), 259-261.

[4] Pendavingh, R., Van Zwam, S., et Al. Sage Matroid Package, included in Sage Mathematics Software 5.11. The Sage Matroid Development Team, 2013. http://www.sagemath.org.

[5] Seymour, P. Decomposition of regular matroids. Journal of Combinatorial Theory, Series B 28, 3 (1980), 305-359.

[6] Stein, W., ET AL. Sage Mathematics Software (Version 5.11). The Sage Development Team, 2013. http://www.sagemath.org.

[7] Tutre, W. Connectivity in matroids. Canadian Journal of Mathematics 18 (1966), 1301-1324.

[8] Whittle, G. Stabilizers of classes of representable matroids. Journal of Combinatorial Theory, Series B 7r, 1 (1999), 39-72. 